Tohoku Math. J.

56 (2004), 501-522

\title{
A GENERALIZATION OF ROBERTS' COUNTEREXAMPLE TO THE FOURTEENTH PROBLEM OF HILBERT
}

\author{
SHIGERU KURODA \\ (Received February 28, 2003, revised July 17, 2003)
}

\begin{abstract}
We generalize Roberts' counterexample to the fourteenth problem of Hilbert, and give a sufficient condition for certain invariant rings not to be finitely generated. It shows that there exist a lot of counterexamples of this type. We also determine the initial algebra of Roberts' counterexample for some monomial order.
\end{abstract}

1. Introduction. The fourteenth problem of Hilbert asks whether the $K$-algebra $L \cap A$ is finitely generated. Here, $K$ is a field, $A$ is a polynomial ring over $K$, and $L$ is a subfield of the quotient field of $A$ containing $K$. The first counterexample to this problem was found by Nagata in 1958. It was given as the invariant subring of a polynomial ring in 32 variables for a linear action of the 13-dimensional additive group (cf. [12]). Recently, Mukai [11] showed that there exists a similar counterexample which is the invariant subring of a polynomial ring in 18 variables for a linear action of the three-dimensional additive group.

In 1990, Roberts gave a simple new counterexample of different type as follows.

THEOREM 1.1 (Roberts [14, Theorem 1]). Let $A=K\left[x_{1}, x_{2}, x_{3}, y_{1}, y_{2}, y_{3}, y_{4}\right]$ be $a$ polynomial ring in seven variables over a field $K$ of characteristic zero. For each nonnegative integer $t$, let $L_{t}$ be the subfield of the quotient field of A generated by

$$
x_{1}, \quad x_{2}, \quad x_{3}, \quad x_{1} y_{4}-x_{2}^{t} x_{3}^{t} y_{1}, \quad x_{2} y_{4}-x_{1}^{t} x_{3}^{t} y_{2}, \quad x_{3} y_{4}-x_{1}^{t} x_{2}^{t} y_{3}
$$

over $K$. If $t \geq 2$, then the $K$-algebra $L_{t} \cap A$ is not finitely generated.

Following this result, Deveney and Finston [2] showed that this counterexample can be obtained as the invariant subring of $A$ for a nonlinear action of the one-dimensional additive group $G_{a}$. Kojima and Miyanishi [6] generalized Roberts' counterexample. They constructed a $G_{a}$-invariant subring of the polynomial ring of each dimension greater than or equal to seven which is not finitely generated. Furthermore, Freudenburg [4] gave a counterexample in dimension six, while Daigle and Freudenburg [1] gave one in dimension five.

In the present paper, we will generalize Roberts' counterexample further, and show that there exist a lot of counterexamples of this type. We give in Theorems 1.3 and 1.4 sufficient conditions for a certain kind of $G_{a}$-invariant subring of a polynomial ring not to be finitely generated. In Section 3, we will discuss Roberts' counterexample $L_{t} \cap A$ in terms of the theory

2000 Mathematics Subject Classification. Primary 13A50; Secondary 13E15, 13N15, 13P10, 14R20.

Partly supported by the Grant-in-Aid for JSPS Fellows, The Ministry of Education, Science, Sports and Culture, Japan. 
of SAGBI (Subalgebra Analogue to Gröbner Bases for Ideals) bases. As a consequence, we determine a generating set of it in Theorem 3.3. We also remark on a sufficient condition for finite generation in Section 4.

Throughout this paper, let $K$ denote a field of characteristic zero. Assume that $R$ is a commutative $K$-algebra, and $A$ is a commutative $R$-algebra. An $R$-homomorphism $D: A \rightarrow$ $A$ is called an $R$-derivation on $A$ if $D(a b)=D(a) b+a D(b)$ holds for any $a, b \in A$. Then, its kernel

$$
A^{D}=\{a \in A \mid D(a)=0\}
$$

is an $R$-subalgebra of $A$. An $R$-derivation $D$ on $A$ is said to be locally nilpotent if, for each $a \in A$, there exists $r \in \boldsymbol{Z}_{\geq 0}$ such that $D^{r}(a)=0$. Here, we denote by $\boldsymbol{Z}_{\geq 0}$ the set of nonnegative integers. We remark that a locally nilpotent $R$-derivation $D$ on $A$ defines an action $A \rightarrow A \otimes_{R} R[t]$ of the one-dimensional additive group scheme $G_{a}=\operatorname{Spec} R[t]$ over $R$ on $A$ by $a \mapsto \sum_{k \geq 0} D^{k}(a) \otimes\left(t^{k} / k !\right)$. The invariant subring $A^{G_{a}}$ of $A$ for this action of $G_{a}$ is equal to $A^{D}$ (cf. [10]).

Let $R=K[x]=K\left[x_{1}, \ldots, x_{m}\right]$ be the polynomial ring in $m$ variables over $K$, and $A=K[\boldsymbol{x}][\boldsymbol{y}]=K[\boldsymbol{x}]\left[y_{1}, \ldots, y_{n}\right]$ that in $n$ variables over $K[\boldsymbol{x}]$. A $K[\boldsymbol{x}]$-derivation $D$ on $K[\boldsymbol{x}][\boldsymbol{y}]$ is said to be elementary if $D\left(y_{j}\right)$ is in $K[x]$ for each $j$. Note that an elementary $K[x]$-derivation is locally nilpotent. An elementary $K[x]$-derivation $D$ on $K[x][y]$ is said to be monomial if each $D\left(y_{i}\right)$ is a monomial, i.e., $x_{1}^{a_{1}} \cdots x_{m}^{a_{n}}$ for some $\left(a_{1}, \ldots, a_{m}\right) \in\left(\boldsymbol{Z}_{\geq 0}\right)^{m}$. In this paper, we discuss the problem of finite generation of the kernel $K[\boldsymbol{x}][\boldsymbol{y}]^{D}$ of an elementary monomial $K[\boldsymbol{x}]$-derivation $D$. As we remarked above, it is equal to the invariant subring of $K[\boldsymbol{x}][\boldsymbol{y}]$ for an action of $G_{a}$, since $D$ is locally nilpotent. Note that $K[\boldsymbol{x}][\boldsymbol{y}]^{D}$ is finitely generated over $K$ if and only if it is so over $K[\boldsymbol{x}]$.

In the case of $n=m+1$, the $K[\boldsymbol{x}]$-derivation

$$
D_{t, m}=x_{1}^{t+1} \frac{\partial}{\partial y_{1}}+\cdots+x_{m}^{t+1} \frac{\partial}{\partial y_{m}}+\left(x_{1} \cdots x_{m}\right)^{t} \frac{\partial}{\partial y_{m+1}}
$$

on $K[\boldsymbol{x}][\boldsymbol{y}]$ is elementary and monomial. The kernel $K[\boldsymbol{x}][\boldsymbol{y}]^{D_{t, m}}$ of this $K[\boldsymbol{x}]$-derivation has been studied well. Deveney and Finston [2] showed that Roberts' $K$-algebra $L_{t} \cap A$ in Theorem 1.1 is equal to the kernel $K[\boldsymbol{x}][\boldsymbol{y}]^{D_{t, m}}$ for $m=3$ (see also Maubach's result found in [3, Section 9.6]). Furthermore, Kojima and Miyanishi showed the following.

THEOREM 1.2 (Kojima-Miyanishi [6]). Assume that $n=m+1$. If $t \geq 2$ and $m \geq 3$, then the kernel $K[\boldsymbol{x}][\boldsymbol{y}]^{D_{t, m}}$ of the $K[\boldsymbol{x}]$-derivation $D_{t, m}$ is not finitely generated over $K$.

We will study the kernel $K[\boldsymbol{x}][\boldsymbol{y}]^{D}$ of an elementary monomial $K[\boldsymbol{x}]$-derivation $D$ on $K[\boldsymbol{x}][\boldsymbol{y}]$ of more general form. Let $D\left(y_{i}\right)=\boldsymbol{x}^{\delta_{i}}$ for each $i=1, \ldots, n$. Here, we denote by $\boldsymbol{x}^{a}$ the monomial $x_{1}^{a_{1}} \cdots x_{m}^{a_{m}}$ for $a=\left(a_{1}, \ldots, a_{m}\right) \in \boldsymbol{Z}^{m}$. Similarly, we denote by $\boldsymbol{y}^{b}$ the monomial $y_{1}^{b_{1}} \ldots y_{n}^{b_{n}}$ for $b=\left(b_{1}, \ldots, b_{n}\right) \in Z^{n}$. Put $\varepsilon_{i, j}=\delta_{i}-\delta_{j}$ for $i, j$, and for $k=1, \ldots, m$, let $\varepsilon_{i, j}^{k}$ and $\delta_{i}^{k}$ be the $k$-th components of $\varepsilon_{i, j}$ and $\delta_{i}$, respectively.

In Sections 1 and 2, we deal with the case where $n \geq 4, m \geq n-1$ and $\varepsilon_{i, j}^{i}>0$ for any $1 \leq i \leq n-1,1 \leq j \leq n$ with $i \neq j$. The derivation $D_{t, m}$ satisfies this condition with 
$\varepsilon_{i, j}^{i}=t+1$ if $j \neq m+1$, and $\varepsilon_{i, j}^{i}=1$ otherwise. We define

$$
\eta=\frac{\varepsilon_{1, n}^{1}}{\min \left\{\varepsilon_{1, j}^{1} \mid j=2, \ldots, n-1\right\}},
$$

and

$$
\eta_{k, i}=\eta \min \left\{\max \left\{\varepsilon_{1, k}^{i}, \varepsilon_{2, k}^{i}\right\}, 0\right\}
$$

for $i=2, \ldots, n-1$ and $k=3, \ldots, n-1$. For each $k=3, \ldots, n-1$, we set $\mathcal{L}_{k, n-2}$ to be the system of linear inequalities

$$
\left\{\begin{array}{l}
u_{1}+\cdots+u_{n-2}=1 \\
u_{1} \geq \eta, u_{i} \geq 0(i=2, \ldots, n-2) \\
\sum_{j=1}^{n-2} \min \left\{\varepsilon_{n, 1}^{i}, \varepsilon_{n, j+1}^{i}\right\} u_{j}+\eta_{k, i} \geq 0(i=2, \ldots, n-1)
\end{array}\right.
$$

in the $n-2$ variables $u_{1}, \ldots, u_{n-2}$.

Here is our main result.

THEOREM 1.3. Assume that $n \geq 4, m \geq n-1$ and $\varepsilon_{i, j}^{i}>0$ for any $1 \leq i \leq n-1$, $1 \leq j \leq n$ with $i \neq j$. If the system $\mathcal{L}_{k, n-2}$ of linear inequalities has a solution in $\boldsymbol{R}^{n-2}$ for each $k=3, \ldots, n-1$, then $K[x][y]^{D}$ is not finitely generated over $K$.

By this theorem, we get the following simple criterion for $n=4$.

THEOREM 1.4. Assume that $m \geq 3, n=4$ and $\varepsilon_{i, j}^{i}>0$ for any $1 \leq i \leq 3,1 \leq j \leq 4$ with $i \neq j$. If

$$
\frac{\varepsilon_{1,4}^{1}}{\min \left\{\varepsilon_{1,2}^{1}, \varepsilon_{1,3}^{1}\right\}}+\frac{\varepsilon_{2,4}^{2}}{\min \left\{\varepsilon_{2,3}^{2}, \varepsilon_{2,1}^{2}\right\}}+\frac{\varepsilon_{3,4}^{3}}{\min \left\{\varepsilon_{3,1}^{3}, \varepsilon_{3,2}^{3}\right\}} \leq 1,
$$

then $K[\boldsymbol{x}][\boldsymbol{y}]^{D}$ is not finitely generated over $K$.

The examples of Roberts are included as special cases of this theorem for $m=3$. In case $(m, n)=(3,4)$, there exist 2450001 derivations on $K[x][y]$ which satisfy (1.6) and $\operatorname{gcd}\left\{\boldsymbol{x}^{\delta_{1}}, \boldsymbol{x}^{\delta_{2}}, \boldsymbol{x}^{\delta_{3}}, \boldsymbol{x}^{\delta_{4}}\right\}=1$ even if we impose the restriction $\delta_{i}^{k} \leq 10$ for all $i, k$.

In the following corollary, the case where $m \geq 4$ and $t=1$ is new, while the case $m \geq 3$ and $t \geq 2$ was proved in [6].

COROLLARY 1.5. Assume that $n=m+1$. If $m \geq 3$ and $t \geq 2$, or $m \geq 4$ and $t=1$, then the kernel $K[x][y]^{D_{t, m}}$ of the $K[x]$-derivation $D_{t, m}$ is not finitely generated over $K$.

We will prove Theorems 1.3, 1.4 and Corollary 1.5 in Section 2.

We remark that, if $t=0$, then the kernel $K[x][y]^{D_{t, m}}$ of $D_{t, m}$ is finitely generated for any $m$ by Weitzenböck's theorem (cf. [12, Chapter IV]). In fact, it is isomorphic to a polynomial ring in $2 m$ variables over $K$ by the remark after Lemma 4.2 below. If $m \leq 2$, then $K[\boldsymbol{x}][y]^{D_{t, m}}$ is also isomorphic to a polynomial ring in $2 m$ variables over $K$ for any $t \geq 0$ 
by [5, Theorem 3.1]. For $(t, m)=(1,3)$, Kurano [7] showed that $K[\boldsymbol{x}][\boldsymbol{y}]^{D_{t, m}}$ is generated by nine elements over $K[\boldsymbol{x}]$.

The author would like to thank Professor Masanori Ishida for helpful comments and encouragement. He also thanks Professor Kazuhiko Kurano for informing him of the result on the kernel of $D_{1,3}$.

2. Construction of invariants. In this section, we prove Theorem 1.3 , and show Theorem 1.4 and Corollary 1.5 as its consequences. Throughout this section, we assume that $n \geq$ 4, $m \geq n-1$ and that $D$ satisfies $\varepsilon_{i, j}^{i}>0$ for any $1 \leq i \leq n-1,1 \leq j \leq n$ with $i \neq j$. We denote $K\left[\boldsymbol{x}, x_{n}^{-1}, \ldots, x_{m}^{-1}\right][\boldsymbol{y}]=K[\boldsymbol{x}][\boldsymbol{y}] \otimes_{K\left[x_{n}, \ldots, x_{m}\right]} K\left[x_{n}, \ldots, x_{m}, x_{n}^{-1}, \ldots, x_{m}^{-1}\right]$. Note that $D$ is uniquely extended to a $K[\boldsymbol{x}]$-derivation on each $K[\boldsymbol{x}]$-subalgebra of $K\left[\boldsymbol{x}, \boldsymbol{x}^{-1}\right][\boldsymbol{y}]$.

Theorem 1.3 follows from the following two lemmas.

LEMMA 2.1. If a monomial of the form $\boldsymbol{x}^{a} y_{n}^{l}$ with $l>0$ appears in an element of $K[\boldsymbol{x}][\boldsymbol{y}]^{D}$, then at least one of the first $n-1$ components of $a \in\left(\boldsymbol{Z}_{\geq 0}\right)^{m}$ is positive.

PROOF. Suppose to the contrary that there appears in $f \in K[\boldsymbol{x}][\boldsymbol{y}]^{D}$ a monomial $x^{a} y_{n}^{l}$ with the first $n-1$ components of $a$ zero with nonzero coefficient. Then, the monomial $\boldsymbol{x}^{a} \boldsymbol{x}^{\delta_{n}} y_{n}^{l-1}$ appears in $D(f)$. Since $D(f)=0$, its coefficient in $D(f)$ is zero. Hence, $\boldsymbol{x}^{a} \boldsymbol{x}^{\delta_{n}} y_{n}^{l-1}$ appears as a monomial in $D\left(\boldsymbol{x}^{a^{\prime}} \boldsymbol{y}^{b^{\prime}}\right)$ for some monomial $\boldsymbol{x}^{a^{\prime}} \boldsymbol{y}^{b^{\prime}} \neq \boldsymbol{x}^{a} y_{n}^{l}$ of $f$. Such $\boldsymbol{x}^{a^{\prime}} \boldsymbol{y}^{b^{\prime}}$ must be equal to $\boldsymbol{x}^{a} \boldsymbol{x}^{\varepsilon_{n, i}} y_{i} y_{n}^{l-1}$ for some $i<n$. Since $\varepsilon_{n, i}^{i}<0$ for $i<n$, we have $\boldsymbol{x}^{a^{\prime}} \boldsymbol{y}^{b^{\prime}} \notin K[\boldsymbol{x}][\boldsymbol{y}]$. This contradicts $f \in K[\boldsymbol{x}][\boldsymbol{y}]$. Thus, at least one of the first $n-1$ components of $a \in\left(\boldsymbol{Z}_{\geq 0}\right)^{m}$ is positive.

The lemma below asserts the existence of an infinite system of invariants.

LEMMA 2.2. Under the assumption in Theorem 1.3, there exists a positive integer $\alpha$ such that a Laurent polynomial of the form

$$
x_{1}^{\alpha} y_{n}^{l}+\left(\text { terms of lower degree in } y_{n}\right)
$$

belongs to $K\left[\boldsymbol{x}, x_{n}^{-1}, \ldots, x_{m}^{-1}\right][\boldsymbol{y}]^{D}$ for each $l>0$.

First, we show Theorem 1.3 by assuming these lemmas. Suppose that $K[\boldsymbol{x}][\boldsymbol{y}]^{D}$ is generated by a finite number of elements $g_{1}, \ldots, g_{p}$. Then, by Lemma 2.1, there exists $r>0$ such that each monomial appearing in $g_{i}$ of the form $x_{1}^{\beta} \boldsymbol{x}^{b} y_{n}^{l}$ with $l>0$ and the first $n-1$ components of $b$ zero satisfies $l / \beta<r$ for every $i$. Since every element of $K[\boldsymbol{x}][\boldsymbol{y}]^{D}$ is written as a sum of products of $g_{1}, \ldots, g_{p}$, a monomial appearing in an element of $K[\boldsymbol{x}][\boldsymbol{y}]^{D}$ is a product of monomials contained in $g_{1}, \ldots, g_{p}$. Hence, any monomial appearing in an element of $K[\boldsymbol{x}][\boldsymbol{y}]^{D}$ of the form $x_{1}^{\beta} \boldsymbol{x}^{b} y_{n}^{l}$ with $l>0$ and the first $n-1$ components of $b$ zero also satisfies $l / \beta<r$. By Lemma 2.2, there appears in some $f \in K\left[\boldsymbol{x}, x_{n}^{-1}, \ldots, x_{m}^{-1}\right][\boldsymbol{y}]^{D}$ a monomial $x_{1}^{\alpha} y_{n}^{l}$ with $l / \alpha>r$. Since $\boldsymbol{x}^{a} f$ is in $K[\boldsymbol{x}][\boldsymbol{y}]^{D}$ for some $a \in\left(\boldsymbol{Z}_{\geq 0}\right)^{m}$ whose first $n-1$ components are zero, we are led to a contradiction. Thus, $K[\boldsymbol{x}][\boldsymbol{y}]^{D}$ is not finitely generated. 
Let us denote by $K[\boldsymbol{y}]_{l}$ the $K$-vector subspace of $K[\boldsymbol{y}]=K\left[y_{1}, \ldots, y_{n}\right]$ of homogeneous $l$-forms in $y_{1}, \ldots, y_{n}$. For each $f=\sum_{b \in Z^{n}} \lambda_{b} \boldsymbol{y}^{b} \in K[\boldsymbol{y}]$, we define the support $\operatorname{supp}(f)$ of $f$ by

$$
\operatorname{supp}(f)=\left\{b \in \boldsymbol{Z}^{n} \mid \lambda_{b} \neq 0\right\} .
$$

For each $a \in \boldsymbol{Z}^{m}$, we define the $K$-linear map $\tau_{\boldsymbol{x}^{a}}: K[\boldsymbol{y}] \rightarrow K\left[\boldsymbol{x}, \boldsymbol{x}^{-1}\right][\boldsymbol{y}]$ by $\tau_{\boldsymbol{x}^{a}}\left(\boldsymbol{y}^{b}\right)=$ $\boldsymbol{x}^{a^{\prime}} \boldsymbol{y}^{b}$. Here, $b=\left(b_{1}, \ldots, b_{n}\right)$ and $a^{\prime}=a+\sum_{j=1}^{n} b_{j} \varepsilon_{n, j}$. We define an elementary $K$ derivation $E$ on $K[y]$ by

$$
E=\frac{\partial}{\partial y_{1}}+\cdots+\frac{\partial}{\partial y_{n}} .
$$

Then, it follows that $D\left(\tau_{\boldsymbol{x}^{a}}(f)\right)=\boldsymbol{x}^{\delta_{n}} \tau_{\boldsymbol{x}^{a}}(E(f))$ for each $a \in \boldsymbol{Z}^{m}$ and $f \in K[\boldsymbol{y}]$. We set

$$
B=K\left[y_{2}-y_{1}, y_{3}-y_{1}, \ldots, y_{n}-y_{1}\right] .
$$

Then, $\tau_{\boldsymbol{x}^{a}}(B) \subset K\left[\boldsymbol{x}, \boldsymbol{x}^{-1}\right][\boldsymbol{y}]^{D}$ for $a \in \boldsymbol{Z}^{m}$. Actually, $D\left(\tau_{\boldsymbol{x}^{a}}(f)\right)=\boldsymbol{x}^{\delta_{n}} \tau_{\boldsymbol{x}^{a}}(E(f))=0$ for $f \in B$, since $E(f)=0$. We define $\boldsymbol{R}$-linear maps $l_{i}: \boldsymbol{R}^{n} \rightarrow \boldsymbol{R}$ by

$$
l_{1}\left(\left(b_{1}, \ldots, b_{n}\right)\right)=\varepsilon_{n, 1}^{1} b_{1}+\min \left\{\varepsilon_{n, j}^{1} \mid j=2, \ldots, n-1\right\} \sum_{j=2}^{n-1} b_{j}
$$

and

$$
l_{i}\left(\left(b_{1}, \ldots, b_{n}\right)\right)=\sum_{j=1}^{n-1} \min \left\{\varepsilon_{n, 1}^{i}, \varepsilon_{n, j}^{i}\right\} b_{j}
$$

for $i=2, \ldots, n-1$. We put $B_{l}=B \cap K[\boldsymbol{y}]_{l}$ for each $l \in \boldsymbol{Z}_{\geq 0}$.

We reduce Lemma 2.2 to the following lemma.

LEMMA 2.3. Under the assumption in Theorem 1.3, there exists a positive integer $\alpha$ such that, for each positive integer $l$, we may find $f \in B_{l}$ such that $(0, \ldots, 0, l) \in \operatorname{supp}(f)$ and every $b \in \operatorname{supp}(f)$ satisfies $l_{1}(b)+\alpha \geq 0$ and $l_{i}(b) \geq 0$ for $i=2, \ldots, n-1$.

Lemma 2.2 is proved by this lemma as follows. As we mentioned above, $\tau_{x_{1}^{\alpha}}(f)$ is in $K\left[\boldsymbol{x}, \boldsymbol{x}^{-1}\right][\boldsymbol{y}]^{D}$. It has the form of (2.1). We show that it is in $K\left[\boldsymbol{x}, x_{n}^{-1}, \ldots, x_{m}^{-1}\right][\boldsymbol{y}]$. By definition, every monomial appearing in $\tau_{x_{1}^{\alpha}}(f)$ is written as $x_{1}^{\alpha} x^{a^{\prime}} \boldsymbol{y}^{b}$, where $b=\left(b_{1}, \ldots, b_{n}\right) \in$ $\operatorname{supp}(f)$ and $a^{\prime}=\sum_{j=1}^{n} b_{j} \varepsilon_{n, j}$. By assumption, we have

$$
\sum_{j=1}^{n} b_{j} \varepsilon_{n, j}^{1}+\alpha \geq l_{1}(b)+\alpha \geq 0
$$

and

$$
\sum_{j=1}^{n} b_{j} \varepsilon_{n, j}^{i} \geq l_{i}(b) \geq 0
$$


for $i=2, \ldots, n-1$. Hence, $x_{1}^{\alpha} \boldsymbol{x}^{a^{\prime}} \boldsymbol{y}^{b}$ does not have negative power in $x_{1}, \ldots, x_{n-1}$. Thus, $\tau_{x_{1}^{\alpha}}(f)$ is in $K\left[\boldsymbol{x}, x_{n}^{-1}, \ldots, x_{m}^{-1}\right][\boldsymbol{y}]^{D}$. This proves Lemma 2.2.

Let $P_{D}$ be the set of $b=\left(b_{1}, \ldots, b_{n}\right) \in\left(\boldsymbol{R}_{\geq 0}\right)^{n}$ with

$$
b_{1}=b_{n}=0, \quad b_{2}+\cdots+b_{n-1}=1, \quad l_{i}(b) \geq 0 \quad(i=2, \ldots, n-1) .
$$

Here, we denote by $\boldsymbol{R}_{\geq 0}$ the set of nonnegative real numbers. For each $b=\left(b_{1}, \ldots, b_{n-2}\right) \in$ $\boldsymbol{R}^{n-2}$, we set $\iota(b)=\left(0, b_{1}, \ldots, b_{n-2}, 0\right)$. Note that, if $b \in\left(\boldsymbol{R}_{\geq 0}\right)^{n-2}$ is a solution of $\mathcal{L}_{k, n-2}$, then $l_{i}(\iota(b))+\eta_{k, i} \geq 0$ for $i=2, \ldots, n-1$. This condition is equivalent to the condition that $\iota(b), \iota(b)+\eta\left(\boldsymbol{e}_{k}-\boldsymbol{e}_{2}\right) \in P_{D}$, where $\boldsymbol{e}_{1}, \ldots, \boldsymbol{e}_{n}$ are the coordinate unit vectors of $\boldsymbol{R}^{n}$. Indeed, if $\varepsilon_{n, k}^{i}<\varepsilon_{n, 1}^{i}$, then

$$
\begin{aligned}
\eta_{k, i} & =\eta \min \left\{\max \left\{\varepsilon_{1, k}^{i}, \varepsilon_{2, k}^{i}\right\}, 0\right\} \\
& =\eta \min \left\{\varepsilon_{n, k}^{i}-\min \left\{\varepsilon_{n, 1}^{i}, \varepsilon_{n, 2}^{i}\right\}, 0\right\} \\
& =\eta \min \left\{\min \left\{\varepsilon_{n, k}^{i}, \varepsilon_{n, 1}^{i}\right\}-\min \left\{\varepsilon_{n, 1}^{i}, \varepsilon_{n, 2}^{i}\right\}, 0\right\} \\
& =\min \left\{\eta l_{i}\left(\boldsymbol{e}_{k}-\boldsymbol{e}_{2}\right), 0\right\} .
\end{aligned}
$$

If $\varepsilon_{n, k}^{i} \geq \varepsilon_{n, 1}^{i}$, then $\varepsilon_{1, k}^{i} \geq 0$. The equality $\eta_{k, i}=\min \left\{\eta l_{i}\left(\boldsymbol{e}_{k}-\boldsymbol{e}_{2}\right), 0\right\}$ also holds in this case, since the right hand sides of the first and the third equality in (2.8) are zero.

For a convex subset $P \subset \boldsymbol{R}^{n}$, we denote $r P=\{r b \mid b \in P\}$ for $r \in \boldsymbol{R}_{\geq 0}$.

LEMMA 2.4. Under the assumption in Theorem 1.3, there exists $\alpha^{\prime}>0$ such that, for any $r>\alpha^{\prime}$ and $u_{3}, \ldots, u_{n-1} \geq 0$ with $\sum_{k=3}^{n-1} u_{k} \leq \eta\left(r-\alpha^{\prime}\right)$, there exist $p_{3}, \ldots, p_{n-1} \in Z_{\geq 0}$ such that

$$
r \boldsymbol{e}_{2}+\sum_{k=3}^{n-1}\left(s_{k} u_{k}+p_{k}\right)\left(\boldsymbol{e}_{k}-\boldsymbol{e}_{2}\right) \in r P_{D}
$$

for any $s_{3}, \ldots, s_{n-1} \in[0,1]$

Proof. Since $\mathcal{L}_{k, n-2}$ has a solution, there exists $\boldsymbol{b}_{k} \in P_{D}$ with $\boldsymbol{b}_{k}+\eta\left(\boldsymbol{e}_{k}-\boldsymbol{e}_{2}\right) \in P_{D}$ for each $k=3, \ldots, n-1$. Let $P$ be the convex hull of

$$
\left\{\boldsymbol{b}_{k}, \boldsymbol{b}_{k}+\eta\left(\boldsymbol{e}_{k}-\boldsymbol{e}_{2}\right) \mid k=3, \ldots, n-1\right\}
$$

in $\boldsymbol{R}^{n}$, and $d$ a positive number such that the $d$-neighborhood of a point $\boldsymbol{a} \in P$ is contained in $P$. Here, we consider the Euclidean topology induced from that on the affine subspace $H=\boldsymbol{e}_{2}+\sum_{k=3}^{n-1} \boldsymbol{R}\left(\boldsymbol{e}_{k}-\boldsymbol{e}_{2}\right)$. Then, define $\alpha^{\prime}=(1 / d) \sqrt{(n-2)(n-3)}$. We show that this $\alpha^{\prime}$ satisfies the desired property.

Take any $r>\alpha^{\prime}$. Note that it suffices to show (2.9) for $u_{3}, \ldots, u_{n-1} \geq 0$ with $\sum_{k=3}^{n-1} u_{k}=$ $\eta\left(r-\alpha^{\prime}\right)$. We set $u_{k}^{\prime}=u_{k} /\left(\eta\left(r-\alpha^{\prime}\right)\right)$ for each $k$. Then,

$$
\sum_{k=3}^{n-1} u_{k}^{\prime}\left(\boldsymbol{b}_{k}+s_{k} \eta\left(\boldsymbol{e}_{k}-\boldsymbol{e}_{2}\right)\right) \in P
$$


for any $s_{3}, \ldots, s_{n-1} \in[0,1]$. Actually, since $P$ is convex,

$$
\boldsymbol{b}_{k}+s_{k} \eta\left(\boldsymbol{e}_{k}-\boldsymbol{e}_{2}\right)=\left(1-s_{k}\right) \boldsymbol{b}_{k}+s_{k}\left(\boldsymbol{b}_{k}+\eta\left(\boldsymbol{e}_{k}-\boldsymbol{e}_{2}\right)\right)
$$

is in $P$ for each $k$. Since $\sum_{k=3}^{n-1} u_{k}^{\prime}=1$, we get (2.10).

For each $\boldsymbol{q} \in H$, define a map $T_{\boldsymbol{q}}: P \rightarrow r H$ by $T_{\boldsymbol{q}}(c)=\alpha^{\prime} \boldsymbol{q}+\left(r-\alpha^{\prime}\right) c$. Since $0<\alpha^{\prime}<r$, we have $T_{\boldsymbol{q}}(P) \subset r P$ if $\boldsymbol{q} \in P$. Put $\boldsymbol{b}^{\prime}=T_{\boldsymbol{a}}\left(\sum_{k=3}^{n-1} u_{k}^{\prime} \boldsymbol{b}_{k}\right)$, and choose $p_{k}^{\prime} \in \boldsymbol{R}_{\geq 0}$ so that $\boldsymbol{b}^{\prime}=r \boldsymbol{e}_{2}+\sum_{k=3}^{n-1} p_{k}^{\prime}\left(\boldsymbol{e}_{k}-\boldsymbol{e}_{2}\right)$. Then, let $p_{k}$ be the nonnegative integer we obtain by adding an element in $(-1 / 2,1 / 2]$ to $p_{k}^{\prime}$ for each $k$. Put $\boldsymbol{b}=r \boldsymbol{e}_{2}+\sum_{k=3}^{n-1} p_{k}\left(\boldsymbol{e}_{k}-\boldsymbol{e}_{2}\right)$ and $\boldsymbol{a}^{\prime}=\boldsymbol{a}+\left(\alpha^{\prime}\right)^{-1}\left(\boldsymbol{b}-\boldsymbol{b}^{\prime}\right)$. Then,

$$
\left|\boldsymbol{b}-\boldsymbol{b}^{\prime}\right|=\sqrt{\left(\sum_{k=3}^{n-1}\left(p_{k}-p_{k}^{\prime}\right)\right)^{2}+\sum_{k=3}^{n-1}\left(p_{k}-p_{k}^{\prime}\right)^{2}} \leq \frac{\sqrt{(n-2)(n-3)}}{2} .
$$

So, we have

$$
\left|\boldsymbol{a}-\boldsymbol{a}^{\prime}\right|=\left(\alpha^{\prime}\right)^{-1}\left|\boldsymbol{b}-\boldsymbol{b}^{\prime}\right| \leq d / 2 .
$$

By the choice of $\boldsymbol{a}$, the point $\boldsymbol{a}^{\prime}$ is in $P$. Hence, $T_{\boldsymbol{a}^{\prime}}(P) \subset r P$. Moreover,

$$
T_{\boldsymbol{a}^{\prime}}(c)-T_{\boldsymbol{a}}(c)=\alpha^{\prime}\left(\boldsymbol{a}^{\prime}-\boldsymbol{a}\right)=\boldsymbol{b}-\boldsymbol{b}^{\prime}
$$

for $c \in P$. Thus, we get

$$
\left(\boldsymbol{b}-\boldsymbol{b}^{\prime}\right)+T_{\boldsymbol{a}}(P) \subset r P
$$

On the other hand, we have

$$
\begin{aligned}
\left(\boldsymbol{b}-\boldsymbol{b}^{\prime}\right)+T_{\boldsymbol{a}}\left(\sum_{k=3}^{n-1} u_{k}^{\prime}\left(\boldsymbol{b}_{k}+s_{k} \eta\left(\boldsymbol{e}_{k}-\boldsymbol{e}_{2}\right)\right)\right) & =\boldsymbol{b}+\sum_{k=3}^{n-1} s_{k} u_{k}\left(\boldsymbol{e}_{k}-\boldsymbol{e}_{2}\right) \\
& =r \boldsymbol{e}_{2}+\sum_{k=3}^{n-1}\left(p_{k}+s_{k} u_{k}\right)\left(\boldsymbol{e}_{k}-\boldsymbol{e}_{2}\right) .
\end{aligned}
$$

It is in $\left(\boldsymbol{b}-\boldsymbol{b}^{\prime}\right)+T_{\boldsymbol{a}}(P)$ for any $s_{k} \in[0,1]$ by (2.10). Then, (2.9) follows from (2.11), since $r P$ is contained in $r P_{D}$. Therefore, $\alpha^{\prime}$ satisfies the desired property.

Now, let us prove Lemma 2.3. First, we show that the assumption that each $\mathcal{L}_{k, n-2}$ has a solution implies that $\varepsilon_{n, 1}^{i} \geq 0$ and $\varepsilon_{n, i}^{1}>0$ for $i=2, \ldots, n-1$. Suppose to the contrary that $\varepsilon_{n, 1}^{i}<0$ for some $2 \leq i \leq n-1$. Then, for any $\left(u_{1}, \ldots, u_{n-2}\right) \in\left(\boldsymbol{R}_{\geq 0}\right)^{n-2}$ with $\sum_{j=1}^{n-2} u_{j}=1$, we have

$$
\sum_{j=1}^{n-2} \min \left\{\varepsilon_{n, 1}^{i}, \varepsilon_{n, j+1}^{i}\right\} u_{j}+\eta_{k, i} \leq \varepsilon_{n, 1}^{i}+\eta_{k, i}<0 .
$$

This contradicts the assumption that $\mathcal{L}_{k, n-2}$ has a solution. Thus, $\varepsilon_{n, 1}^{i} \geq 0$ for $i=2, \ldots$, $n-1$. Suppose that $\varepsilon_{n, i}^{1} \leq 0$ for some $2 \leq i \leq n-1$. Then, it implies that $\eta \geq 1$, since

$$
\varepsilon_{1, n}^{1}-\min \left\{\varepsilon_{1, j}^{1} \mid j=2, \ldots, n-1\right\}=-\min \left\{\varepsilon_{n, j}^{1} \mid j=2, \ldots, n-1\right\} \geq-\varepsilon_{n, i}^{1} \geq 0 .
$$


If $\mathcal{L}_{k, n-2}$ has a solution $u=\left(u_{1}, \ldots, u_{n-2}\right)$, then $\eta=u_{1}=1$ and $u_{j}=0$ for $j=2, \ldots$, $n-2$. For this $u$, it follows that

$$
\sum_{j=1}^{n-2} \min \left\{\varepsilon_{n, 1}^{2}, \varepsilon_{n, j+1}^{2}\right\} u_{j}+\eta_{k, 2}=\min \left\{\varepsilon_{n, 1}^{2}, \varepsilon_{n, 2}^{2}\right\}+\eta_{k, 2} \leq \varepsilon_{n, 2}^{2}<0 .
$$

This is a contradiction. Thus, $\varepsilon_{n, i}^{1}>0$ for $i=2, \ldots, n-1$.

Take $\alpha^{\prime}>0$ as in Lemma 2.4, and set $\alpha$ to be an integer greater than or equal to $\alpha^{\prime} \varepsilon_{1, n}^{1}$. Let $l$ be an arbitrary positive integer, and $\mathcal{F}$ the set of $f \in B_{l}$ such that $(0, \ldots, 0, l) \in \operatorname{supp}(f)$ and every $b \in \operatorname{supp}(f)$ satisfies $l_{i}(b) \geq 0$ for $i=2, \ldots, n-1$. Since

$$
l_{i}\left(j \boldsymbol{e}_{1}+(l-j) \boldsymbol{e}_{n}\right)=j \varepsilon_{n, 1}^{i} \geq 0
$$

for $i=2, \ldots, n-1$ and $j=0, \ldots, l$, we have $\left(y_{n}-y_{1}\right)^{l} \in \mathcal{F}$. Hence, $\mathcal{F} \neq \varnothing$. We show that there exists $F_{0} \in \mathcal{F}$ such that $l_{1}(b)+\alpha \geq 0$ for each $b \in \operatorname{supp}\left(F_{0}\right)$. Suppose the contrary. Then, for each $f \in \mathcal{F}$, an element $O(f)=(d, e)$ in $Z^{2}$ is defined by setting $d$ to be the maximum among the $n$-th components of $b \in \operatorname{supp}(f)$ with $l_{1}(b)+\alpha<0$, and $e$ to be the maximum among the first components of $b \in \operatorname{supp}(f)$ whose $n$-th components are $d$. We define the total order $\preceq$ on $Z^{2}$ by $\left(d_{1}, e_{1}\right) \preceq\left(d_{2}, e_{2}\right)$ if $d_{1}<d_{2}$ or $d_{1}=d_{2}, e_{1} \leq e_{2}$. For $v_{1}, v_{2} \in Z^{2}$, we denote $v_{1} \prec v_{2}$ if $v_{1} \preceq v_{2}$ and $v_{1} \neq v_{2}$. Choose $F \in \mathcal{F}$ with $O(F)=(d, e)$ such that $(d, e) \preceq O(h)$ for any $h \in \mathcal{F}$, and set $f \in K\left[y_{2}, \ldots, y_{n-1}\right]$ to be the coefficient of $y_{1}^{e} y_{n}^{d}$ in $F$.

For $b \in \operatorname{supp}(F)$ whose first and $n$-th components are $e$ and $d$, respectively, we have

$$
\begin{aligned}
l_{1}(b)+\alpha & =\varepsilon_{n, 1}^{1} e+\min \left\{\varepsilon_{n, j}^{1} \mid j=2, \ldots, n-1\right\}(l-d-e)+\alpha \\
& =\varepsilon_{n, 1}^{1} e+\left(\varepsilon_{n, 1}^{1}+\min \left\{\varepsilon_{1, j}^{1} \mid j=2, \ldots, n-1\right\}\right)(l-d-e)+\alpha \\
& =\min \left\{\varepsilon_{1, j}^{1} \mid j=2, \ldots, n-1\right\}(l-d-e)-\varepsilon_{1, n}^{1}(l-d)+\alpha \\
& \geq \min \left\{\varepsilon_{1, j}^{1} \mid j=2, \ldots, n-1\right\}(l-d-e)-\varepsilon_{1, n}^{1}\left(l-d-\alpha^{\prime}\right) \\
& =\min \left\{\varepsilon_{1, j}^{1} \mid j=2, \ldots, n-1\right\}\left((l-d-e)-\eta\left(l-d-\alpha^{\prime}\right)\right) .
\end{aligned}
$$

Since $\varepsilon_{1, j}^{1}>0$ for $j \neq 1$, the right hand side of the third equality in (2.12) is negative by the maximality of $e$. By the last equality in (2.12) we get

$$
l-d-e<\eta\left(l-d-\alpha^{\prime}\right) .
$$

LEMMA 2.5. In the above notation, $E(f)=0$.

Proof. Suppose that $E(f) \neq 0$. Let $\boldsymbol{y}^{b}$ be a monomial appearing in $E(f)$ with nonzero coefficient. Let $\lambda_{j}^{\prime}$ be the coefficient of $y_{j} \boldsymbol{y}^{b}$ in $f$, and $b_{j}$ the $j$-th component of $b$ for each $j$. Then, the coefficient $\mu^{\prime}$ of $\boldsymbol{y}^{b}$ in $E(f)$ is written as

$$
\mu^{\prime}=\sum_{j=2}^{n-1}\left(b_{j}+1\right) \lambda_{j}^{\prime}
$$


Let $\lambda_{j}$ be the coefficient of $y_{j} \boldsymbol{y}^{b}\left(y_{1}^{e} y_{n}^{d}\right)$ in $F$ for each $j$. Then, $\lambda_{j}=\lambda_{j}^{\prime}$ for $j=2, \ldots, n-1$. The coefficient $\mu$ of $\boldsymbol{y}^{b}\left(y_{1}^{e} y_{n}^{d}\right)$ in $E(F)$ is written as

$$
\mu=(e+1) \lambda_{1}+\sum_{j=2}^{n-1}\left(b_{j}+1\right) \lambda_{j}+(d+1) \lambda_{n}=(e+1) \lambda_{1}+\mu^{\prime}+(d+1) \lambda_{n} .
$$

Since $E(F)=0$, we have $\mu=0$. Moreover, $\lambda_{1}=0$ by the maximality of $e$. Since $\mu^{\prime} \neq 0$, we have $\lambda_{n} \neq 0$, that is,

$$
b^{\prime}=b+e \boldsymbol{e}_{1}+(d+1) \boldsymbol{e}_{n}
$$

is in $\operatorname{supp}(F)$. Note that $l_{1}\left(b^{\prime}+\boldsymbol{e}_{2}-\boldsymbol{e}_{n}\right)+\alpha$ is negative, since it is equal to the left hand side of the first equality in (2.12). Hence,

$$
\begin{aligned}
l_{1}\left(b^{\prime}\right)+\alpha & =l_{1}\left(b^{\prime}+\boldsymbol{e}_{2}-\boldsymbol{e}_{n}\right)+\alpha+l_{1}\left(\boldsymbol{e}_{n}-\boldsymbol{e}_{2}\right) \\
& <l_{1}\left(\boldsymbol{e}_{n}-\boldsymbol{e}_{2}\right)=-\min \left\{\varepsilon_{n, j}^{1} \mid j=2, \ldots, n-1\right\}<0 .
\end{aligned}
$$

This contradicts the maximality of $d$. Thus, we get $E(f)=0$.

We claim that $K[y]^{E} \subset B$. This is a special case of Lemma 4.2 which we shall prove later. By Lemma 2.5, this fact implies that $f$ is in $B_{l-d-e}$.

LEMMA 2.6. In the above notation, there exists $G \in B_{l}$ of the form $G=f y_{1}^{e} y_{n}^{d}+g$, where $g \in K[y]_{l}$ such that every $b \in \operatorname{supp}(g)$ satisfies the following. $l_{i}(b) \geq 0$ for $i=$ $2, \ldots, n-1$. If $e^{\prime}$ and $d^{\prime}$ are the first and $n$-th components of $b$, respectively, then $\left(d^{\prime}, e^{\prime}\right) \prec$ $(d, e)$.

ProOF. Since $f$ is in $B_{l-d-e} \cap K\left[y_{2}, \ldots, y_{n-1}\right]$, we have

$$
f=\sum_{u} \lambda_{u} \prod_{k=3}^{n-1}\left(y_{2}-y_{k}\right)^{u_{k}}
$$

for some $\lambda_{u} \in K$. Here, the sum in the equality above is taken over $u=\left(u_{3}, \ldots, u_{n-1}\right) \in$ $\left(\boldsymbol{Z}_{\geq 0}\right)^{n-3}$ with $\sum_{k=3}^{n-1} u_{k}=l-d-e$. By (2.13), we get $\sum_{k=3}^{n-1} u_{k}<\eta\left(l-d-\alpha^{\prime}\right)$ for each $u$. Hence, there exist $p_{3}, \ldots, p_{n-1} \in \boldsymbol{Z}_{\geq 0}$ such that

$$
(l-d) \boldsymbol{e}_{2}+\sum_{k=3}^{n-1}\left(s_{k} u_{k}+p_{k}\right)\left(\boldsymbol{e}_{k}-\boldsymbol{e}_{2}\right) \in(l-d) P_{D}
$$

for any $s_{3}, \ldots, s_{n-1} \in[0,1]$ by Lemma 2.4 . We set

$$
h_{u}^{\prime}=y_{2}^{e-p} \prod_{k=3}^{n-1}\left(\left(y_{2}-y_{k}\right)^{u_{k}} y_{k}^{p_{k}}\right)
$$

where $p=\sum_{k=3}^{n-1} p_{k}$. Note that each element of $\operatorname{supp}\left(h_{u}^{\prime}\right)$ is written as the left hand side of (2.14) for some $s_{3}, \ldots, s_{n-1} \in[0,1]$. So, $\operatorname{supp}\left(h_{u}^{\prime}\right)$ is contained in $(l-d) P_{D}$. In particular, 
$e-p \geq 0$. We set

$$
h_{u}=\left(y_{1}-y_{2}\right)^{e-p} \prod_{k=3}^{n-1}\left(\left(y_{2}-y_{k}\right)^{u_{k}}\left(y_{1}-y_{k}\right)^{p_{k}}\right)
$$

for each $u$, and define

$$
G=\left(\sum_{u} \lambda_{u} h_{u}\right)\left(y_{n}-y_{1}\right)^{d} .
$$

Put $g=G-f y_{1}^{e} y_{n}^{d}$. Then, the first and $n$-th components $e^{\prime}$ and $d^{\prime}$, respectively, of each $b \in \operatorname{supp}(g)$ satisfy $\left(d^{\prime}, e^{\prime}\right) \prec(d, e)$. So, we verify that $l_{i}(b) \geq 0$ for $i=2, \ldots, n-1$ for each $b \in \operatorname{supp}(g)$. Each element of $\operatorname{supp}\left(h_{u}\right)$ is contained in $c+\sum_{j=2}^{n-1} \boldsymbol{Z}_{\geq 0}\left(\boldsymbol{e}_{1}-\boldsymbol{e}_{j}\right)$ for some $c \in(l-d) P_{D}$. Indeed, $h_{u}$ is equal to the polynomial obtained from $h_{u}^{\prime}$ by substituting $y_{1}-y_{k}$ for $y_{k}$ for each $k$, and $\operatorname{supp}\left(h_{u}^{\prime}\right) \subset(l-d) P_{D}$. Therefore, we may write each $b \in \operatorname{supp}(g)$ as

$$
b=d_{1} \boldsymbol{e}_{1}+d_{2} \boldsymbol{e}_{n}+c+\sum_{j=2}^{n-1} v_{j}\left(\boldsymbol{e}_{1}-\boldsymbol{e}_{j}\right),
$$

where $d_{1}, d_{2}, v_{2}, \ldots, v_{n-1} \in \boldsymbol{Z}_{\geq 0}$ and $c \in(l-d) P_{D}$. Note that $l_{i}\left(\boldsymbol{e}_{n}\right)=0$ and $l_{i}\left(\boldsymbol{e}_{1}\right), l_{i}(c) \geq$ 0 for $i=2, \ldots, n-1$. Moreover,

$$
\begin{aligned}
l_{i}\left(\sum_{j=2}^{n-1} v_{j}\left(\boldsymbol{e}_{1}-\boldsymbol{e}_{j}\right)\right) & =-\sum_{j=2}^{n-1} \min \left\{\varepsilon_{n, 1}^{i}, \varepsilon_{n, j}^{i}\right\} v_{j}+\min \left\{\varepsilon_{n, 1}^{i}, \varepsilon_{n, 1}^{i}\right\} \sum_{j=2}^{n-1} v_{j} \\
& =\sum_{j=2}^{n-1}\left(\varepsilon_{n, 1}^{i}-\min \left\{\varepsilon_{n, 1}^{i}, \varepsilon_{n, j}^{i}\right\}\right) v_{j} \geq 0 .
\end{aligned}
$$

Thus, we get $l_{i}(b) \geq 0$ for $i=2, \ldots, n-1$.

We set $H=F-G$. Then, $H$ is in $\mathcal{F}$. Moreover, $O(H) \prec O(F)$ by the definition of $H$. This contradicts the choice of $F$. Hence, there exists $F_{0} \in \mathcal{F}$ such that $l_{1}(b)+\alpha \geq 0$ for each $b \in \operatorname{supp}\left(F_{0}\right)$. We have thus proved Lemma 2.3. Therefore, the proof of Theorem 1.3 is completed.

Now, assume that $m \geq 3$ and $n=4$. Then, we set

$$
\xi_{i}=\xi_{i}(D)=\frac{\varepsilon_{i, 4}^{i}}{\min \left\{\varepsilon_{i, j}^{i}, \varepsilon_{i, k}^{i}\right\}}
$$

for distinct integers $1 \leq i, j, k \leq 3$, and put $\xi(D)=\xi_{1}(D)+\xi_{2}(D)+\xi_{3}(D)$.

We show Theorem 1.4 as a consequence of Theorem 1.3. We verify that $\left(1-\xi_{2}, \xi_{2}\right)$ is a solution of $\mathcal{L}_{3,2}$. Note that $\xi_{i}>0$ for $i=1,2,3, \eta=\xi_{1}, \eta_{3,2}=0$ and $\eta_{3,3}=$ $-\xi_{1} \min \left\{\varepsilon_{3,1}^{3}, \varepsilon_{3,2}^{3}\right\}$. So, $\xi_{2}>0$. By (1.6), we have $1-\xi_{2} \geq \xi_{1}+\xi_{3}>\xi_{1}=\eta$. Moreover, it 
follows that

$$
\begin{aligned}
& \min \left\{\varepsilon_{4,1}^{2}, \varepsilon_{4,2}^{2}\right\}\left(1-\xi_{2}\right)+\min \left\{\varepsilon_{4,1}^{2}, \varepsilon_{4,3}^{2}\right\} \xi_{2}+\eta_{3,2} \\
& \quad=\min \left\{\varepsilon_{4,1}^{2}, \varepsilon_{4,2}^{2}\right\}+\left(\min \left\{\varepsilon_{4,1}^{2}, \varepsilon_{4,3}^{2}\right\}-\min \left\{\varepsilon_{4,1}^{2}, \varepsilon_{4,2}^{2}\right\}\right) \xi_{2}+\eta_{3,2} \\
& \quad=\varepsilon_{4,2}^{2}+\min \left\{\varepsilon_{2,1}^{2}, \varepsilon_{2,3}^{2}\right\} \xi_{2}=0
\end{aligned}
$$

and

$$
\begin{aligned}
\min & \left\{\varepsilon_{4,1}^{3}, \varepsilon_{4,2}^{3}\right\}\left(1-\xi_{2}\right)+\min \left\{\varepsilon_{4,1}^{3}, \varepsilon_{4,3}^{3}\right\} \xi_{2}+\eta_{3,3} \\
& =\min \left\{\varepsilon_{4,1}^{3}, \varepsilon_{4,2}^{3}\right\}+\left(\min \left\{\varepsilon_{4,1}^{3}, \varepsilon_{4,3}^{3}\right\}-\min \left\{\varepsilon_{4,1}^{3}, \varepsilon_{4,2}^{3}\right\}\right) \xi_{2}+\eta_{3,3} \\
& =\left(\varepsilon_{4,3}^{3}+\min \left\{\varepsilon_{3,1}^{3}, \varepsilon_{3,2}^{3}\right\}\right)-\min \left\{\varepsilon_{3,1}^{3}, \varepsilon_{3,2}^{3}\right\} \xi_{2}+\eta_{3,3} \\
& =\min \left\{\varepsilon_{3,1}^{3}, \varepsilon_{3,2}^{3}\right\}\left(-\xi_{3}+1-\xi_{2}-\xi_{1}\right) \geq 0 .
\end{aligned}
$$

Therefore, $\left(1-\xi_{2}, \xi_{2}\right)$ is a solution of $\mathcal{L}_{3,2}$. Hence, $K[\boldsymbol{x}][\boldsymbol{y}]^{D}$ is not finitely generated by Theorem 1.3.

Finally, we show Corollary 1.5. As mentioned in Section $1, \varepsilon_{i, j}^{i}>0$ for any $i \neq j$, since $\varepsilon_{i, j}^{i}=t+1$ if $j \neq m+1$, and $\varepsilon_{i, j}^{i}=1$ otherwise. Assume that $m=3$ and $t \geq 2$. Then, $\xi\left(D_{t, m}\right)=3 /(t+1) \leq 1$. Hence, $K[\boldsymbol{x}][\boldsymbol{y}]^{D_{t, 3}}$ is not finitely generated by Theorem 1.4.

Assume that $m \geq 4$ and $t \geq 1$. For $k=3, \ldots, m-1$, we define $u_{k}=\left(u_{k}^{1}, \ldots, u_{k}^{m-1}\right) \in$ $\left(\boldsymbol{R}_{\geq 0}\right)^{m-1}$ as follows. Set $u_{3}^{3}, u_{k}^{j}=1 / 2$ for $j, k$ with $j=1$ or $k=j+2$, and set $u_{k}^{j}=$ 0 otherwise. We show that $u_{k}$ is a solution of $\mathcal{L}_{k, m-1}$ for each $k$. Since $m \geq 4$, we have $\sum_{j=1}^{m-1} u_{k}^{j}=1$. Since $t \geq 1$, we get $u_{k}^{1}=1 / 2 \geq 1 /(t+1)=\eta$. Clearly, $u_{k}^{j} \geq 0$ for $j=2, \ldots, m-1$. For $i=2, \ldots, m-1$, it follows that

$$
\sum_{j=1}^{m-1} \min \left\{\varepsilon_{m+1,1}^{i}, \varepsilon_{m+1, j+1}^{i}\right\} u_{k}^{j}+\eta_{k, i}=t-(t+1) u_{k}^{i-1}+\eta_{k, i} .
$$

Note that $\eta_{k, i}=-1$ if $i=k$, and $\eta_{k, i}=0$ otherwise. If $i=k$, then the right hand side of (2.16) is equal to $t-1$, since $u_{k}^{k-1}=0$. If $i \neq k$, then it is not less than $(t-1) / 2$, since $u_{k}^{i-1} \leq 1 / 2$ for any $i, k$. So, it is nonnegative for every $i, k$. Therefore, $u_{k}$ is a solution of $\mathcal{L}_{k, m-1}$ for $k=3, \ldots, m-2$. By Theorem $1.3, K[\boldsymbol{x}]^{D_{t, m}}$ is not finitely generated. Thus, we complete the proof of Corollary 1.5 .

3. A SAGBI basis for the counterexample of Roberts. In this section, we consider the counterexample of Roberts. Recall that it is obtained as the kernel of the derivation $D_{t, m}$ on $K[\boldsymbol{x}][\boldsymbol{y}]$ for $(m, n)=(3,4)$ and $t \geq 2$ by the result of Deveney and Finston [2]. We determine its initial algebra for some monomial order on $K[\boldsymbol{x}][\boldsymbol{y}]$. Consequently, it will turn out that the infinite system of invariants appearing in Roberts' proof of [14, Lemma 3] is a generating set of $K[\boldsymbol{x}][\boldsymbol{y}]^{D_{t, 3}}$.

First, we review the notion of an initial algebra and a SAGBI (Subalgebra Analogue to Gröbner Bases for Ideals) basis. Let $\preceq$ be a monomial order on $K[\boldsymbol{x}][\boldsymbol{y}]$, i.e., a total order on $\boldsymbol{Z}^{m} \times \boldsymbol{Z}^{n}$ such that $a \preceq b$ implies $a+c \preceq b+c$ for any $a, b, c \in \boldsymbol{Z}^{m} \times \boldsymbol{Z}^{n}$ and the zero 
vector is the minimum among $\left(\boldsymbol{Z}_{>0}\right)^{m} \times\left(\boldsymbol{Z}_{>0}\right)^{n}$ for $\preceq$. We denote $a \prec b$ if $a \neq b$ and $a \preceq b$. We sometimes denote $\boldsymbol{x}^{a} \boldsymbol{y}^{b} \preceq \boldsymbol{x}^{a^{\prime}} \boldsymbol{y}^{b^{\prime}}$ instead of $(a, b) \preceq\left(a^{\prime}, b^{\prime}\right)$. For $f \in K[\boldsymbol{x}][\boldsymbol{y}] \backslash\{0\}$, we define the initial term $\operatorname{in}_{\leq}(f)$ of $f$ by $\alpha \boldsymbol{x}^{a} \boldsymbol{y}^{b}$. Here, $(a, b)$ is the maximal element of $\operatorname{supp}(f)$ for $\preceq$, and $\alpha$ is the coefficient of $\boldsymbol{x}^{a} \boldsymbol{y}^{b}$ in $f$. Note that the maximum of $\operatorname{supp}(f)$ always exists, since it is a nonempty finite set. If $f=0$, then we define $\operatorname{in}_{\preceq}(f)=0$. Then, it follows that

$$
\operatorname{in}_{\preceq}(f g)=\operatorname{in}_{\preceq}(f) \operatorname{in}_{\preceq}(g)
$$

for any $f, g \in K[x][y]$. Assume that $A$ is a $K$-subalgebra of $K[x][y]$. We define the initial algebra $\operatorname{in}_{\leq}(A)$ of $A$ as the $K$-vector space generated by $\left\{\operatorname{in}_{\leq}(f) \mid f \in A\right\}$. Then, $\operatorname{in}_{\leq}(A)$ is a $K$-algebra by (3.1). We say that a generating set $\mathcal{S}$ of $A$ is a SAGBI basis if the initial algebra $\operatorname{in}_{\leq}(A)$ is generated by $\left\{\operatorname{in}_{\leq}(f) \mid f \in \mathcal{S}\right\}$ over $K$.

The following is a basic property of a SAGBI basis.

Lemma 3.1 (Robbiano-Sweedler [13, Proposition 1.16]). Let $\preceq$ be a monomial order on $K[\boldsymbol{x}][\boldsymbol{y}]$. Assume that $A$ is a $K$-subalgebra of $K[\boldsymbol{x}][\boldsymbol{y}]$, and $\mathcal{S}$ is a subset of $A$. If $\left\{\mathrm{in}_{\leq}(f) \mid\right.$ $f \in \mathcal{S}\}$ generates the initial algebra $\operatorname{in}_{\leq}(A)$ over $K$, then $\mathcal{S}$ is a SAGBI basis for $A$. In particular, $\mathcal{S}$ generates A over $K$.

For any elementary monomial $K[x]$-derivation $D$ on $K[x][y]$, we set $\varepsilon_{i, j}^{+}$to be the vector we obtain from $\varepsilon_{i, j}$ by replacing the negative components by zero, and define $L_{i, j}=\boldsymbol{x}^{\varepsilon_{j, i}^{+}} y_{i}-$ $\boldsymbol{x}^{\varepsilon_{i, j}^{+}} y_{j}$ for each $i, j$. Then, $L_{i, j}$ is in $K[\boldsymbol{x}][\boldsymbol{y}]^{D}$ for $i, j$.

Now, let us consider the kernel $K[\boldsymbol{x}][\boldsymbol{y}]^{D_{t, m}}$ of $D_{t, m}$ on $K[\boldsymbol{x}][\boldsymbol{y}]$ for $(m, n)=(3,4)$. Note that the three elements

$$
x_{1}^{t+1} y_{2}-x_{2}^{t+1} y_{1}, \quad x_{1}^{t+1} y_{3}-x_{3}^{t+1} y_{1}, \quad x_{2}^{t+1} y_{3}-x_{3}^{t+1} y_{2}
$$

are contained in $K[\boldsymbol{x}][\boldsymbol{y}]^{D_{t, 3}}$. Indeed, they are equal to $L_{2,1}, L_{3,1}$ and $L_{3,2}$. Moreover, we know the following (see also [6, Lemma 2.1]).

THEOREM 3.2 (Roberts [14, Lemma 3]). For each $d \in Z_{>0}$ and $i=1,2,3$, there exists an element of the form $x_{i} y_{4}^{d}+\left(\right.$ terms of lower degree in $\left.y_{4}\right)$ in $K[\boldsymbol{x}][\boldsymbol{y}]^{D_{t, 3}}$.

We take an arbitrary $I_{d, i} \in K[\boldsymbol{x}][\boldsymbol{y}]^{D_{t, 3}}$ of the form in Theorem 3.2 for each $(d, i)$. Note that $I_{0, i}=x_{i}$ for each $i$. Let $\preceq_{\text {lex }}$ be the monomial order on $K[\boldsymbol{x}][\boldsymbol{y}]$ for $(m, n)=(3,4)$ which is the lexicographic order with

$$
x_{1} \prec_{\text {lex }} x_{2} \prec_{\text {lex }} x_{3} \prec_{\text {lex }} y_{1} \prec_{\text {lex }} y_{2} \prec_{\text {lex }} y_{3} \prec_{\text {lex }} y_{4} \text {. }
$$

Namely, we define $a \preceq_{\operatorname{lex}} b$ if the last nonzero component of $b-a$ is positive for $a, b \in$ $\boldsymbol{Z}^{3} \times \boldsymbol{Z}^{4}$, where we regard $a, b$ as elements of $\boldsymbol{Z}^{7}$.

The following is the main result of this section.

THEOREM 3.3. Assume that $t \geq 2$. Then, the initial algebra of $K[x][y]^{D_{t, 3}}$ for $\preceq_{\text {lex }}$ is generated by

$$
\left\{x_{1}^{t+1} y_{2}, x_{1}^{t+1} y_{3}, x_{2}^{t+1} y_{3}\right\} \cup\left\{x_{i} y_{4}^{d} \mid d \in Z_{\geq 0}, i=1,2,3\right\}
$$

over $K$. The set 


$$
\left\{x_{1}^{t+1} y_{2}-x_{2}^{t+1} y_{1}, x_{1}^{t+1} y_{3}-x_{3}^{t+1} y_{1}, x_{2}^{t+1} y_{3}-x_{3}^{t+1} y_{2}\right\} \cup\left\{I_{d, i} \mid d \in \boldsymbol{Z}_{\geq 0}, i=1,2,3\right\}
$$

is a SAGBI basis for $K[\boldsymbol{x}][\boldsymbol{y}]^{D_{t, 3}}$ for $\preceq_{\text {lex. }}$. In particular, it generates $K[\boldsymbol{x}][\boldsymbol{y}]^{D_{t, 3}}$ over $K$.

To analyze $K[x][y]^{D}$ in greater detail, we define a grading structure on it. Let $D$ be any elementary monomial $K[\boldsymbol{x}]$-derivation on $K[\boldsymbol{x}][\boldsymbol{y}]$. We set

$$
\Gamma=\left(\boldsymbol{Z}^{m} \times \boldsymbol{Z}^{n}\right) / \sum_{i=2}^{n} \boldsymbol{Z}\left(\varepsilon_{i, 1}, \boldsymbol{e}_{1}-\boldsymbol{e}_{i}\right),
$$

and $K\left[\boldsymbol{x}, \boldsymbol{x}^{-1}\right][\boldsymbol{y}]_{\gamma}$ the $K$-vector space generated by monomials $\boldsymbol{x}^{a} \boldsymbol{y}^{b}$ for $(a, b) \in$ $\boldsymbol{Z}^{m} \times\left(\boldsymbol{Z}_{\geq 0}\right)^{n}$ with the image of $(a, b)$ in $\Gamma$ equal to $\gamma$ for each $\gamma \in \Gamma$. Then, it defines a $\Gamma$-grading on $K\left[\boldsymbol{x}, \boldsymbol{x}^{-1}\right][\boldsymbol{y}]$, i.e., $K\left[\boldsymbol{x}, \boldsymbol{x}^{-1}\right][\boldsymbol{y}]=\bigoplus_{\gamma \in \Gamma} K\left[\boldsymbol{x}, \boldsymbol{x}^{-1}\right][\boldsymbol{y}]_{\gamma}$ and $K\left[\boldsymbol{x}, \boldsymbol{x}^{-1}\right][\boldsymbol{y}]_{\gamma} K\left[\boldsymbol{x}, \boldsymbol{x}^{-1}\right][\boldsymbol{y}]_{\mu} \subset K\left[\boldsymbol{x}, \boldsymbol{x}^{-1}\right][\boldsymbol{y}]_{\gamma+\mu}$ for any $\gamma, \mu \in \Gamma$. Moreover, it follows that

$$
K\left[\boldsymbol{x}, \boldsymbol{x}^{-1}\right][\boldsymbol{y}]^{D}=\bigoplus_{\gamma \in \Gamma} K\left[\boldsymbol{x}, \boldsymbol{x}^{-1}\right][\boldsymbol{y}]_{\gamma}^{D} .
$$

Here, for a $K$-subalgebra $A$ of $K\left[\boldsymbol{x}, \boldsymbol{x}^{-1}\right][\boldsymbol{y}]$, we set $A_{\gamma}=A \cap K\left[\boldsymbol{x}, \boldsymbol{x}^{-1}\right][\boldsymbol{y}]_{\gamma}$ for each $\gamma$. We say that $f \in K\left[\boldsymbol{x}, \boldsymbol{x}^{-1}\right][\boldsymbol{y}]$ is $\Gamma$-homogeneous if $f$ is in $K\left[\boldsymbol{x}, \boldsymbol{x}^{-1}\right][\boldsymbol{y}]_{\gamma}$ for some $\gamma \in \Gamma$. This $\gamma$ is denoted by $\operatorname{deg}_{\Gamma}(f)$. Note that each $\gamma \in \Gamma$ is expressed as the image of $\left(a, l \boldsymbol{e}_{n}\right)$ for some $a \in \boldsymbol{Z}^{m}$ and $l \in \boldsymbol{Z}_{\geq 0}$. Then, we have $\tau_{\boldsymbol{x}^{a}}\left(K[\boldsymbol{y}]_{l}\right)=K\left[\boldsymbol{x}, \boldsymbol{x}^{-1}\right][\boldsymbol{y}]_{\gamma}$. Actually, $\tau_{\boldsymbol{x}^{a}}(\phi(f))=f$ for $f \in K\left[\boldsymbol{x}, \boldsymbol{x}^{-1}\right][\boldsymbol{y}]_{\gamma}$, where $\phi: K\left[\boldsymbol{x}, \boldsymbol{x}^{-1}\right][\boldsymbol{y}] \rightarrow K[\boldsymbol{y}]$ is the homomorphism which substitutes one for each $x_{i}$. Since $E \circ \phi=\phi \circ D$, we have $\phi(f) \in K[\boldsymbol{y}]_{l}^{E}=B_{l}$ for $f \in K\left[\boldsymbol{x}, \boldsymbol{x}^{-1}\right][\boldsymbol{y}]_{\gamma}^{D}$. Hence, $\tau_{\boldsymbol{x}^{a}}\left(B_{l}\right)=K\left[\boldsymbol{x}, \boldsymbol{x}^{-1}\right][\boldsymbol{y}]_{\gamma}^{D}$.

We remark that, for $f \in K[\boldsymbol{y}], r \in \boldsymbol{Z}_{\geq 0}$ and $a \in \boldsymbol{Z}^{m}$, the condition that $\left(y_{i}-y_{j}\right)^{r}$ divides $f$ implies that $L_{i, j}^{r}$ is a factor of $\tau_{x^{a}}(f)$ in $K\left[\boldsymbol{x}, \boldsymbol{x}^{-1}\right][\boldsymbol{y}]$. This is proved as follows. Note that $\tau_{\boldsymbol{x}^{a}}(f)=\boldsymbol{x}^{a} \tau_{1}(f)$ for any $f \in K[\boldsymbol{y}]$, and $\tau_{1}\left(y_{i}-y_{j}\right)=\boldsymbol{x}^{\varepsilon_{n, i}-\varepsilon_{j, i}^{+}} L_{i, j}$ for $i, j$. Assume that $f=\left(y_{i}-y_{j}\right)^{r} f^{\prime}$ for some $f^{\prime} \in K[\boldsymbol{y}]$. Then,

$$
\tau_{x^{a}}(f)=\boldsymbol{x}^{a} \tau_{1}\left(\left(y_{i}-y_{j}\right)^{r} f^{\prime}\right)=\boldsymbol{x}^{a} \tau_{1}\left(y_{i}-y_{j}\right)^{r} \tau_{1}\left(f^{\prime}\right)=\boldsymbol{x}^{a+r\left(\varepsilon_{n, i}-\varepsilon_{j, i}^{+}\right)} L_{i, j}^{r} \tau_{1}\left(f^{\prime}\right),
$$

since $\tau_{1}$ preserves multiplication. Thus, $L_{i, j}^{r}$ is a factor of $\tau_{\boldsymbol{x}^{a}}(f)$ in $K\left[\boldsymbol{x}, \boldsymbol{x}^{-1}\right][\boldsymbol{y}]$.

Assume that $n=3$. Then, each $f \in B_{l}$ is written as

$$
f=\left(y_{2}-y_{1}\right)^{s}\left(y_{3}-y_{1}\right)^{t} \sum_{i=0}^{u} \alpha_{i}\left(y_{2}-y_{1}\right)^{i}\left(y_{3}-y_{1}\right)^{u-i} .
$$

Here, $s, t, u \in \boldsymbol{Z}_{\geq 0}$ with $s+t+u=l$ and $\alpha_{i} \in K$ with $\alpha_{0}, \alpha_{u} \neq 0$. If $\beta_{1}, \ldots, \beta_{u} \in \bar{K}$ are the solutions of the equation $\sum_{i=0}^{u} \alpha_{i} X^{i}=0$, then we get

$$
f=\alpha_{0}\left(y_{2}-y_{1}\right)^{s}\left(y_{3}-y_{1}\right)^{t} \prod_{i=1}^{u}\left(y_{2}-\beta_{i} y_{3}+\left(\beta_{i}-1\right) y_{1}\right),
$$

where $\bar{K}$ is the algebraic closure of $K$. 
Proposition 3.4. Assume that $n=3$, and $D$ is any elementary monomial $K[\boldsymbol{x}]$ derivation on $K[\boldsymbol{x}][\boldsymbol{y}]$. Then,

$$
\left\{x_{1}, \ldots, x_{m}, L_{2,1}, L_{3,1}, L_{3,2}\right\}
$$

is a SAGBI basis for $K[\boldsymbol{x}][\boldsymbol{y}]^{D}$ with respect to any monomial order on $K[\boldsymbol{x}][\boldsymbol{y}]$.

ProOF. Let $\preceq$ be any monomial order on $K[\boldsymbol{x}][\boldsymbol{y}]$. By Proposition 3.1, it suffices to show that $\operatorname{in}_{\leq}\left(K[\boldsymbol{x}][\boldsymbol{y}]^{D}\right)$ is equal to

$$
R=K[\boldsymbol{x}]\left[\mathrm{in}_{\preceq}\left(L_{2,1}\right), \mathrm{in}_{\preceq}\left(L_{3,1}\right), \mathrm{in}_{\preceq}\left(L_{3,2}\right)\right] .
$$

First, we note that, since $\boldsymbol{x}^{a} \tau_{1}\left(y_{i}-y_{j}\right) \in K[\boldsymbol{x}][\boldsymbol{y}]$, its initial term is in $R$ for $a \in \boldsymbol{Z}^{m}$ and $i, j$. Indeed, $\boldsymbol{x}^{a} \tau_{1}\left(y_{i}-y_{j}\right)=\boldsymbol{x}^{a+\varepsilon_{3, i}-\varepsilon_{j, i}^{+}} L_{i, j}$, which is in $K[\boldsymbol{x}][\boldsymbol{y}]$ if and only if $a+$ $\varepsilon_{3, i}-\varepsilon_{j, i}^{+} \in\left(\boldsymbol{Z}_{\geq 0}\right)^{m}$. We show that $\boldsymbol{x}^{a} \tau_{1}(g) \in K[\boldsymbol{x}][\boldsymbol{y}]$ implies that in $\operatorname{in}_{\preceq}\left(\boldsymbol{x}^{a} \tau_{1}(g)\right) \in R \otimes_{K} \bar{K}$ for $a \in \boldsymbol{Z}^{m}$, where $g=y_{2}-y_{1}-\beta\left(y_{3}-y_{1}\right)$ with $\beta \in \bar{K}$. If $\beta$ is zero or one, then we are done. Assume that $\beta \neq 0,1$. Then, there appears in $\boldsymbol{x}^{a} \tau_{1}(g)$ each monomial which appears in $\boldsymbol{x}^{a}\left(\tau_{1}\left(y_{i}-y_{1}\right)\right)$ for $i=2,3$. Hence, if $\boldsymbol{x}^{a} \tau_{1}(g)$ is in $K[\boldsymbol{x}][\boldsymbol{y}]$, then $\boldsymbol{x}^{a} \tau_{1}\left(y_{i}-y_{1}\right)$ is also in $K[\boldsymbol{x}][\boldsymbol{y}]$ for $i=2,3$. Since $\operatorname{in}_{\preceq}\left(\boldsymbol{x}^{a} \tau_{1}(g)\right)$ is equal to $\operatorname{in}_{\preceq}\left(\boldsymbol{x}^{a} \tau_{1}\left(y_{i}-y_{1}\right)\right)$ for some $i \in\{2,3\}$ up to scalar multiplication, it is in $R \otimes_{K} \bar{K}$.

To show $\operatorname{in}_{\preceq}\left(K[\boldsymbol{x}][\boldsymbol{y}]^{D}\right)=R$, it suffices to verify that the initial term $\operatorname{in}_{\preceq}(F)$ of every $\Gamma$-homogeneous element $F \in K[x][y]^{D} \backslash\{0\}$ is in $R$. Put $f=\phi(F)$. Then, it is in $B_{l}$ for some $l \in \boldsymbol{Z}_{\geq 0}$. So, $f$ is expressed as in (3.6). Since $\tau_{\boldsymbol{x}^{a}}(f)=F$ for some $a \in \boldsymbol{Z}^{m}$, we get

$$
F=\tau_{\boldsymbol{x}^{a}}(f)=\alpha_{0} \boldsymbol{x}^{a} \tau_{1}\left(y_{2}-y_{1}\right)^{s} \tau_{1}\left(y_{3}-y_{1}\right)^{t} \prod_{i=1}^{u} \tau_{1}\left(y_{2}-\beta_{i} y_{3}+\left(\beta_{i}-1\right) y_{1}\right) .
$$

Since $F$ is in $K[\boldsymbol{x}][\boldsymbol{y}]$, there exist $a^{\prime}, a^{\prime \prime}, a_{i} \in \boldsymbol{Z}^{m}$ with $s a^{\prime}+t a^{\prime \prime}+\sum_{i=1}^{u} a_{i}=a$ such that $\boldsymbol{x}^{a^{\prime}} \tau_{1}\left(y_{2}-y_{1}\right), \boldsymbol{x}^{a^{\prime \prime}} \tau_{1}\left(y_{3}-y_{1}\right)$ and $\boldsymbol{x}^{a_{i}} \tau_{1}\left(y_{2}-\beta_{i} y_{3}+\left(\beta_{i}-1\right) y_{1}\right)$ are in $K[\boldsymbol{x}][\boldsymbol{y}]$. Hence, their initial terms are in $R \otimes_{K} \bar{K}$, as noted in the preceding paragraph. This implies that $\mathrm{in}_{\preceq}(F) \in R$ by (3.8) and (3.1).

In particular, we have the following.

COROLlary 3.5 (Khoury [5, Corollary 2.2]). Assume that $n=3$, and $D$ is any elementary monomial $K[\boldsymbol{x}]$-derivation on $K[\boldsymbol{x}][\boldsymbol{y}]$. Then,

$$
K[\boldsymbol{x}][\boldsymbol{y}]^{D}=K[\boldsymbol{x}]\left[L_{2,1}, L_{3,1}, L_{3,2}\right] .
$$

As we mentioned before Proposition 3.4, each element $f \in B_{l}$ is factored into the product of $l$ elements in $\bar{K} \otimes_{K} B_{1}$. We note that, if $r$ is the maximal integer such that $\left(y_{3}-y_{2}\right)^{r}$ divides $f$, then the expansion of $f$ involves the monomials $y_{1}^{l-r} y_{2}^{r}, y_{1}^{l-r} y_{3}^{r}$ and does not involve $y_{1}^{l-r^{\prime}} y_{2}^{r^{\prime}}, y_{1}^{l-r^{\prime}} y_{3}^{r^{\prime}}$ for $0 \leq r^{\prime} \leq r$.

Lemma 3.6. Assume that $(m, n)=(3,3)$ and $\varepsilon_{i, j}^{i}>0$ for any $1 \leq i, j \leq 3$ with $i \neq j$. If $\gamma=\operatorname{deg}_{\Gamma}\left(L_{2,1}^{p} L_{3,1}^{q} L_{3,2}^{r}\right)$ for $p, q, r \in \boldsymbol{Z}_{\geq 0}$, then $K[\boldsymbol{x}][\boldsymbol{y}]_{\gamma}^{D}$ is equal to the onedimensional $K$-vector space generated by $L_{2,1}^{p} L_{3,1}^{q} L_{3,2}^{r}$. 
Proof. Take any $0 \neq F \in K[x][y]_{\gamma}^{D}$, and put $f=\phi(F)$. Then, $f$ is in $B_{l}$ and $\tau_{x^{a}}(f)=F$, where $l=p+q+r$ and $a=p\left(\varepsilon_{2,3}+\varepsilon_{1,2}^{+}\right)+q \varepsilon_{1,3}^{+}+r \varepsilon_{2,3}^{+}$. If $\left(y_{2}-y_{1}\right)^{p},\left(y_{3}-y_{1}\right)^{q}$ and $\left(y_{3}-y_{2}\right)^{r}$ divide $f$, then $F$ is in $K\left(L_{2,1}^{p} L_{3,1}^{q} L_{3,2}^{r}\right)$. Actually, it implies that $L_{2,1}^{p}, L_{3,1}^{q}$ and $L_{3,2}^{r}$ are factors of $F$. Suppose, say, that the maximal integer $r^{\prime}$ such that $\left(y_{3}-y_{2}\right)^{r^{\prime}}$ divides $f$ is less than $r$. Then, $y_{1}^{l-r^{\prime}} y_{2}^{r^{\prime}}$ and $y_{1}^{l-r^{\prime}} y_{3}^{r^{\prime}}$ appear in $f$ with nonzero coefficient, as mentioned above. Hence, so do $\tau_{x^{a}}\left(y_{1}^{l-r^{\prime}} y_{2}^{r^{\prime}}\right)$ and $\tau_{\boldsymbol{x}^{a}}\left(y_{1}^{l-r^{\prime}} y_{3}^{r^{\prime}}\right)$ in $F$. By definition, the first component of $\varepsilon_{2,3}^{+}$or $\varepsilon_{3,2}^{+}$is zero. If that of $\varepsilon_{2,3}^{+}$is zero, then the power of $x_{1}$ in $\tau_{x^{a}}\left(y_{1}^{l-r^{\prime}} y_{3}^{r^{\prime}}\right)$ is negative. In fact, $\tau_{x^{a}}\left(y_{1}^{l-r^{\prime}} y_{3}^{r^{\prime}}\right)=\boldsymbol{x}^{a^{\prime}} y_{1}^{l-r^{\prime}} y_{3}^{r^{\prime}}$, where

$$
a^{\prime}=a+\left(l-r^{\prime}\right) \varepsilon_{3,1}=p \varepsilon_{2,1}^{+}+q \varepsilon_{3,1}^{+}+r \varepsilon_{2,3}^{+}-\left(r-r^{\prime}\right) \varepsilon_{1,3} .
$$

Since the first components of $\varepsilon_{2,1}^{+}, \varepsilon_{3,1}^{+}, \varepsilon_{2,3}^{+}$are zero, that of $a^{\prime}$ is equal to $-\left(r-r^{\prime}\right) \varepsilon_{1,3}^{1}<0$. Similarly, the power of $x_{1}$ in $\tau_{\boldsymbol{x}^{a}}\left(y_{1}^{l-r^{\prime}} y_{2}^{r^{\prime}}\right)$ is negative if the first component of $\varepsilon_{3,2}^{+}$is zero. This is a contradiction. Therefore, $F$ is in $K\left(L_{2,1}^{p} L_{3,1}^{q} L_{3,2}^{r}\right)$.

Assume that $n=4$. We define a homomorphism $\tilde{l}: \boldsymbol{Z}^{4} \rightarrow \boldsymbol{Z}$ of additive groups by

$$
\tilde{l}\left(\left(b_{1}, b_{2}, b_{3}, b_{4}\right)\right)=b_{2} \varepsilon_{1,2}^{1}+b_{3} \varepsilon_{1,3}^{1} .
$$

LEMmA 3.7. Assume that $n=4, \varepsilon_{1,2}^{1} \geq \varepsilon_{1,3}^{1}>0$ and $F$ is an element of $B_{l}$ for some $l \in \boldsymbol{Z}_{\geq 0}$. If every $b \in \operatorname{supp}(F)$ satisfies $\tilde{l}(b) \geq p$ for some $p \in \boldsymbol{Z}_{\geq 0}$, then $\left(y_{3}-y_{2}\right)^{q}$ divides $F$ for the minimal $q \in \boldsymbol{Z}_{\geq 0}$ with $p \leq q \varepsilon_{1,3}^{1}$.

ProOF. Write

$$
F=f_{0}\left(y_{4}-y_{1}\right)^{l}+f_{1}\left(y_{4}-y_{1}\right)^{l-1}+\cdots+f_{l},
$$

where $f_{i} \in K\left[y_{2}-y_{1}, y_{3}-y_{1}\right]_{i}$ for each $i$. Suppose that $\left(y_{3}-y_{2}\right)^{q}$ did not divide $F$. Then, there exists $i$ such that $\left(y_{3}-y_{2}\right)^{q}$ does not divide $f_{i}$. Let $i$ be the minimum among such indices $i$, and $q^{\prime}$ the maximal integer such that $\left(y_{3}-y_{2}\right)^{q^{\prime}}$ divides $f_{i}$. Then, $f_{i}$ involves the monomial $y_{1}^{i-q^{\prime}} y_{3}^{q^{\prime}}$, as we noted before Lemma 3.6. We set $b=\left(i-q^{\prime}, 0, q^{\prime}, l-i\right)$. Then, $\tilde{l}(b)=q^{\prime} \varepsilon_{1,3}^{1}<q \varepsilon_{1,3}^{1}$. It implies that $\tilde{l}(b)<p$ by the minimality of $q$. Hence, $b \notin \operatorname{supp}(F)$.

On the other hand, $f_{i}\left(y_{4}-y_{1}\right)^{l-i}$ involves $\boldsymbol{y}^{b}$. If $j>i$, then $f_{j}\left(y_{4}-y_{1}\right)^{l-j}$ does not involve $\boldsymbol{y}^{b}$, since the exponent of $y_{4}$ in each monomial of it is less than $l-i$. Suppose that $f_{j}\left(y_{4}-y_{1}\right)^{l-j}$ involved $\boldsymbol{y}^{b}$ for $j<i$. Then, $f_{j}$ contains $y_{1}^{j-q^{\prime}} y_{3}^{q^{\prime}}$. Since $q^{\prime}<q$, this contradicts the assumption that $\left(y_{3}-y_{2}\right)^{q}$ divides $f_{j}$ by the note above. Therefore, $f_{j}\left(y_{4}-y_{1}\right)^{l-j}$ does not involve $\boldsymbol{y}^{b}$ if $j \neq i$. Hence, $b \in \operatorname{supp}(F)$. This is a contradiction. Therefore, $\left(y_{3}-y_{2}\right)^{q}$ divides $F$.

We remark that, if $F \in K[\boldsymbol{x}][\boldsymbol{y}]^{D}$ is expressed as

$$
F=f_{0} y_{n}^{l}+f_{1} y_{n}^{l-1}+\cdots+f_{l}
$$

for $f_{i} \in K[\boldsymbol{x}]\left[y_{1}, \ldots, y_{n-1}\right]$, then $D\left(f_{0}\right)=0$. Actually, we get

$$
0=D(F)=D\left(f_{0}\right) y_{n}^{l}+\left(\text { terms of lower degree in } y_{n}\right) .
$$


The following is the key proposition.

Proposition 3.8. Assume that $(m, n)=(3,4)$ and $\varepsilon_{i, j}^{i}>0$ for any $1 \leq i, j \leq 4$ with $i \neq j$. Then, the monomial $\boldsymbol{x}^{a} y_{2}^{p} y_{3}^{q+r} y_{4}^{l}$ is not contained in $\operatorname{in}_{\preceq \operatorname{lex}}\left(K[\boldsymbol{x}][\boldsymbol{y}]^{D}\right)$ for any $p, q, r, l \in \boldsymbol{Z}_{\geq 0}$, where we set $a=p \varepsilon_{1,2}^{+}+q \varepsilon_{1,3}^{+}+r \varepsilon_{2,3}^{+}$.

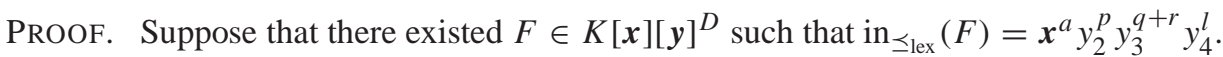
Then, without loss of generality, we may assume that $F$ is $\Gamma$-homogeneous. Write

$$
F=f_{0} y_{4}^{l}+f_{1} y_{4}^{l-1}+\cdots+f_{l},
$$

where $f_{i} \in K[\boldsymbol{x}]\left[y_{1}, y_{2}, y_{3}\right]$ for $i=0, \ldots, l$. Then, $f_{0}$ is in $K[\boldsymbol{x}]\left[y_{1}, y_{2}, y_{3}\right]^{D}$, as we remarked above. Moreover, $f_{0}$ is $\Gamma$-homogeneous and $\operatorname{deg}_{\Gamma}\left(f_{0}\right)=\operatorname{deg}_{\Gamma}\left(L_{1,2}^{p} L_{1,3}^{q} L_{2,3}^{r}\right)$. Hence, $f_{0}$ is equal to $L_{1,2}^{p} L_{1,3}^{q} L_{2,3}^{r}$ up to scalar multiplication by Lemma 3.6.

It suffices to show that each of $L_{2,1}^{p}, L_{3,1}^{q}$ and $L_{3,2}^{r}$ must be a factor of $F$ in $K\left[\boldsymbol{x}, \boldsymbol{x}^{-1}\right][\boldsymbol{y}]$. Indeed, it will imply that $F=L_{1,2}^{p} L_{1,3}^{q} L_{2,3}^{r} F^{\prime}$ for some $F^{\prime} \in K\left[\boldsymbol{x}, \boldsymbol{x}^{-1}\right][\boldsymbol{y}]$, since $L_{2,1}, L_{3,1}$ and $L_{3,2}$ are pairwise prime. Then, $F^{\prime}$ is an element in $K[\boldsymbol{x}][\boldsymbol{y}]^{D}$. However, $F^{\prime}$ involves the monomial $y_{4}^{l}$. This contradicts Lemma 2.1.

Since the arguments are similar, we only show that $L_{3,2}^{r}$ is a factor of $F$. We assume that $\varepsilon_{1,2}^{1} \geq \varepsilon_{1,3}^{1}$. The proof is similar for the other case. We set $f=\phi(F)$, and claim that every $b=\left(b_{1}, b_{2}, b_{3}, b_{4}\right) \in \operatorname{supp}(f)$ satisfies $\tilde{l}(b) \geq r \varepsilon_{1,3}^{1}$. This implies that $\left(y_{3}-y_{2}\right)^{r}$ divides $f$ by Lemma 3.7. Hence, $L_{3,2}^{r}$ is a factor of $F$ in $K\left[\boldsymbol{x}, \boldsymbol{x}^{-1}\right][\boldsymbol{y}]$, and the proof is completed. By straightforward computation, we may verify that $\operatorname{deg}_{\Gamma}(F)$ is equal to the image of $\left(c,(d+l) \boldsymbol{e}_{4}\right)$, where $d=p+q+r$ and

$$
c=p \varepsilon_{2,1}^{+}+q \varepsilon_{3,1}^{+}+r \varepsilon_{2,3}^{+}+d \varepsilon_{1,4}+r \varepsilon_{3,1} .
$$

Thus, it follows that $F=\tau_{\boldsymbol{x}^{c}}(f)$, as mentioned above. Hence, $F$ involves $\tau_{\boldsymbol{x}^{c}}\left(\boldsymbol{y}^{b}\right)$ for $b \in$ $\operatorname{supp}(f)$. By simple computation, we get $\tau_{\boldsymbol{x}^{c}}\left(\boldsymbol{y}^{b}\right)=\boldsymbol{x}^{d} \boldsymbol{y}^{b}$, where

$$
d=p \varepsilon_{2,1}^{+}+q \varepsilon_{3,1}^{+}+r \varepsilon_{2,3}^{+}+\left(l-b_{4}\right) \varepsilon_{4,1}+r \varepsilon_{3,1}+b_{2} \varepsilon_{1,2}+b_{3} \varepsilon_{1,3} .
$$

Note that the first components of $p \varepsilon_{2,1}^{+}, q \varepsilon_{3,1}^{+}, r \varepsilon_{2,3}^{+}$are zero and $b_{4} \leq l$. Since $\boldsymbol{x}^{d} \boldsymbol{y}^{b}$ is in $K[\boldsymbol{x}][\boldsymbol{y}]$, the first component of $d$ is nonnegative. Thus, we have

$$
0 \leq\left(l-b_{4}\right) \varepsilon_{4,1}^{1}+r \varepsilon_{3,1}^{1}+b_{2} \varepsilon_{1,2}^{1}+b_{3} \varepsilon_{1,3}^{1}=\left(l-b_{4}\right) \varepsilon_{4,1}^{1}-r \varepsilon_{1,3}^{1}+\tilde{l}(b) \leq-r \varepsilon_{1,3}^{1}+\tilde{l}(b) .
$$

Therefore, $\tilde{l}(b) \geq r \varepsilon_{1,3}^{1}$.

Now, let us prove Theorem 3.3. By Lemma 3.1, the last statement is a consequence of the first part. So, we will prove the first part.

We set $R$ to be the $K$-algebra generated by (3.4). Clearly, in $\preceq_{\operatorname{lex}}\left(K[\boldsymbol{x}][\boldsymbol{y}]^{D_{t, 3}}\right)$ contains $R$. For the converse, it suffices to show that in $\preceq_{\text {lex }}(F)$ is in $R$ for any $\Gamma$-homogeneous element $F \in K[\boldsymbol{x}][\boldsymbol{y}]^{D_{t, 3}}$. The remark before Proposition 3.8 implies that $\operatorname{in}_{\preceq_{\mathrm{lex}}}(F)=\operatorname{in}_{\preceq_{\mathrm{lex}}}\left(F^{\prime}\right) y_{4}^{l}$ for some $F^{\prime} \in K[\boldsymbol{x}]\left[y_{1}, y_{2}, y_{3}\right]^{D_{t, 3}}$ and $l \in \boldsymbol{Z}_{\geq 0}$. By Proposition 3.4, the set $\left\{x_{1}, x_{2}, x_{3}, L_{2,1}, L_{3,1}\right.$, 
$\left.L_{3,2}\right\}$ is a SAGBI basis for $K[\boldsymbol{x}]\left[y_{1}, y_{2}, y_{3}\right]^{D_{t, 3}}$ with respect to any monomial order. In particular,

$$
\operatorname{in}_{\succeq_{\text {lex }}}\left(K[\boldsymbol{x}]\left[y_{1}, y_{2}, y_{3}\right]^{D_{t, 3}}\right)=K[\boldsymbol{x}]\left[x_{1}^{t+1} y_{2}, x_{1}^{t+1} y_{3}, x_{2}^{t+1} y_{3}\right] .
$$

Hence, there exist $a_{1}, a_{2}, a_{3}, p, q, r \in \boldsymbol{Z}_{\geq 0}$ such that

$$
\operatorname{in}_{\succeq_{\text {lex }}}(F)=\left(x_{1}^{t+1} y_{2}\right)^{p}\left(x_{1}^{t+1} y_{3}\right)^{q}\left(x_{2}^{t+1} y_{3}\right)^{r} x_{1}^{a_{1}} x_{2}^{a_{2}} x_{3}^{a_{3}} y_{4}^{l} \text {. }
$$

Obviously, $\operatorname{in}_{\leq \text {lex }}(F)$ is in $R$ if $l=0$. Assume that $l>0$. Then, $a_{1}+a_{2}+a_{3}>0$ by Proposition 3.8. Hence, it is also in $R$. Therefore, in $\operatorname{in}_{\leq \mathrm{lex}}\left(K[x][y]^{D_{t, 3}}\right)$ is contained in $R$. This completes the proof of Theorem 3.3.

4. A condition for finite generation. In this section, we investigate a condition for the finite generation of $K[x][y]^{D}$, where $D$ is an elementary monomial $K[x]$-derivation. The main result of this section is the following.

THEOREM 4.1. Assume that $(m, n)=(3,4)$, and there exist $i \neq j$ and $k$ such that $\varepsilon_{\tau(i), \tau(j)}^{\sigma(k)} \leq 0$ and $\sigma(k)=\tau(i)$ for every pair of permutations $\sigma$ and $\tau$ on $\{1,2,3\}$ and $\{1,2,3,4\}$, respectively. Then, $K[x][y]^{D}$ is generated by $L_{k_{i}, l_{i}}$ for $i=1,2,3,4$ over $K[x]$ for some integers $1 \leq k_{i}, l_{i} \leq 4$.

First, we look at general properties on the kernel of an elementary monomial $K[x]$ derivation. For each $i, j$, we set $\tilde{L}_{i, j}=y_{i}-\boldsymbol{x}^{\varepsilon_{i, j}} y_{j}$. It is contained in $K\left[\boldsymbol{x}, \boldsymbol{x}^{-1}\right][\boldsymbol{y}]^{D}$. To avoid confusion, we sometimes denote it by $\tilde{L}_{i, j}^{D}$ to emphasize $D$.

LEMmA 4.2. The kernel $K[x][y]^{D}$ is contained in $K[x]\left[\tilde{L}_{1, j}, \ldots, \tilde{L}_{n, j}\right]$ for each $j$.

Proof. Take any $F \in K[x][y]^{D}$, and let $f$ be the polynomial obtained from $F$ by replacing $y_{j}$ by zero. Then, define an element $F^{\prime}$ of $K[x]\left[\tilde{L}_{1, j}, \ldots, \tilde{L}_{n, j}\right]$ as the polynomial which we obtain from $f$ by replacing $y_{k}$ by $\tilde{L}_{k, j}$ for each $k$. We show that $F=F^{\prime}$. Suppose that $F \neq F^{\prime}$. Write

$$
F-F^{\prime}=\left(\text { terms of higher degree in } y_{j}\right)+g y_{j}^{e},
$$

where $g$ is an element of $K\left[\boldsymbol{x}, \boldsymbol{x}^{-1}\right][\boldsymbol{y}] \backslash\{0\}$ not involving $y_{j}$. Since $F-f$ and $F^{\prime}-f$ are in $K\left[\boldsymbol{x}, \boldsymbol{x}^{-1}\right][\boldsymbol{y}] y_{j}$, we have $e>0$. However,

$$
0=D\left(F-F^{\prime}\right)=\left(\text { terms of higher degree in } y_{j}\right)+e g x^{\delta_{j}} y_{j}^{e-1},
$$

a contradiction, since $e g \boldsymbol{x}^{\delta_{j}} \neq 0$. Therefore, $F=F^{\prime}$.

Assume that $\delta_{j}=0$ for some $j$. Then, $\tilde{L}_{k, j}$ is in $K[x][y]^{D}$ for each $k$. By Lemma 4.2, it implies that $K[\boldsymbol{x}][\boldsymbol{y}]^{D}=K[\boldsymbol{x}]\left[\tilde{L}_{1, j}, \ldots, \tilde{L}_{n, j}\right]$. If this is the case, then $K[\boldsymbol{x}][\boldsymbol{y}]^{D}$ is isomorphic to $K[x]\left[y_{1}, \ldots, y_{j-1}, y_{j+1}, \ldots, y_{n}\right]$ via the homomorphism which substitutes zero for $y_{j}$. In particular, the kernel $K[x][y]^{D_{t, m}}$ of the derivation $D_{t, m}$ for $t=0$ is generated by $\tilde{L}_{1, m+1}, \ldots, \tilde{L}_{m, m+1}$ over $K[x]$, and is isomorphic to the polynomial ring in $2 m$ variables over $K$. 
Now, we fix $1 \leq i \leq m$ and $1 \leq j \leq n$. Assume that $\varepsilon_{k, j}^{i} \geq 0$ for every $k=1, \ldots, n$. Then, put $\mu=\min \left\{\varepsilon_{k, j}^{i} \mid k \neq j\right\}$, and set $\boldsymbol{x}^{\varepsilon_{k, j}^{\prime}}=x_{i}^{-\mu} \boldsymbol{x}^{\varepsilon_{k, j}}$ for each $k$. Let $D^{\prime}$ be an elementary monomial $K[\boldsymbol{x}]$-derivation on $K[\boldsymbol{x}][\boldsymbol{y}]$ such that $D^{\prime}\left(y_{k}\right) / D^{\prime}\left(y_{j}\right)=\boldsymbol{x}^{\varepsilon_{k, j}^{\prime}}$ for each $k$. For $f \in K[\boldsymbol{x}][\boldsymbol{y}]^{D}$, we define $T_{j, i}(f)$ to be the polynomial obtained from $f$ by replacing $y_{j}$ by $x_{i}^{-\mu} y_{j}$. Then, it follows that

$$
T_{j, i}\left(\tilde{L}_{k, j}^{D}\right)=y_{k}-\boldsymbol{x}^{\varepsilon_{k, j}}\left(x_{i}^{-\mu} y_{j}\right)=y_{k}-\boldsymbol{x}^{\varepsilon_{k, j}^{\prime}} y_{j}=\tilde{L}_{k, j}^{D^{\prime}}
$$

for each $k$.

LEMMA 4.3. Let $i, j$ be integers with $1 \leq i \leq m$ and $1 \leq j \leq n$. If $\varepsilon_{k, j}^{i} \geq 0$ for every $k=1, \ldots, n$, then $T_{j, i}$ is an injective homomorphism with the image $K[\boldsymbol{x}][\boldsymbol{y}]^{D^{\prime}}$.

Proof. Suppose that $T_{j, i}(f)$ were not in $K[\boldsymbol{x}][\boldsymbol{y}]^{D^{\prime}}$ for some $f \in K[\boldsymbol{x}][\boldsymbol{y}]^{D}$. By Lemma 4.2, $f$ is in $K[x]\left[\left\{\tilde{L}_{k, j}^{D} \mid k\right\}\right]$. Since $T_{j, i}$ sends $\tilde{L}_{k, j}^{D}$ to $\tilde{L}_{k, j}^{D^{\prime}}$, we have $T_{j, i}(f) \in$ $K[\boldsymbol{x}]\left[\left\{\tilde{L}_{k, j}^{D^{\prime}} \mid k\right\}\right]$. In particular, $D^{\prime}\left(T_{j, i}(f)\right)=0$. Hence, there appears in $T_{j, i}(f)$ a monomial with negative power in some variable. By the definition of $T_{j, i}(f)$, the variable must be $x_{i}$. However, $\tilde{L}_{k, j}^{D^{\prime}}$ does not have negative power in $x_{i}$ for each $k$. Hence, such a monomial cannot appear in $T_{j, i}(f)$. This is a contradiction. Thus, $T_{j, i}(f)$ is in $K[\boldsymbol{x}][\boldsymbol{y}]^{D^{\prime}}$.

Conversely, a homomorphism $K[\boldsymbol{x}][\boldsymbol{y}]^{D^{\prime}} \rightarrow K[\boldsymbol{x}][\boldsymbol{y}]^{D}$ is defined by the substitution $y_{j} \mapsto x_{i}^{\mu} y_{j}$. Indeed, it sends each $\tilde{L}_{k, j}^{D^{\prime}}$ to $\tilde{L}_{k, j}^{D}$. It is the inverse of $T_{j, i}: K[\boldsymbol{x}][\boldsymbol{y}]^{D} \rightarrow$ $K[\boldsymbol{x}][\boldsymbol{y}]^{D^{\prime}}$.

We use the following proposition to reduce problems on the kernel of $D$ to a lower dimensional case.

Proposition 4.4. Let $D$ be any elementary monomial $K[\boldsymbol{x}]$-derivation on $K[\boldsymbol{x}][\boldsymbol{y}]$, and $1 \leq j, k \leq m$ distinct integers. For each $1 \leq i \leq m$, we assume that either $\varepsilon_{j, k}^{i} \geq 0$ or $\varepsilon_{l, k}^{i} \geq 0$ for all $l \neq j$. Then,

$$
K[\boldsymbol{x}][\boldsymbol{y}]^{D}=K[\boldsymbol{x}]\left[y_{1}, \ldots, y_{j-1}, y_{j+1}, \ldots, y_{n}\right]^{D}\left[L_{j, k}\right] .
$$

Proof. Clearly, the right hand side of (4.1) is contained in the left hand side. We show the converse. Let $S$ be the set of elements of $K[x][y]^{D}$ not contained in the right hand side of (4.1). Suppose that $S$ were not empty. Take $f \in S$ with the minimal degree in $y_{j}$, and write

$$
f=g_{d}\left(\boldsymbol{x}^{\varepsilon_{k, j}^{+}} y_{j}\right)^{d}+g_{d-1}\left(\boldsymbol{x}^{\varepsilon_{k, j}^{+}} y_{j}\right)^{d-1}+\cdots+g_{0},
$$

where $g_{i} \in K\left[\boldsymbol{x}, \boldsymbol{x}^{-1}\right]\left[y_{1}, \ldots, y_{j-1}, y_{j+1}, \ldots, y_{n}\right]$ with $g_{d} \neq 0$. To complete the proof, it suffices to show that $g_{d}$ is in $K[\boldsymbol{x}][\boldsymbol{y}]^{D}$. Indeed, it implies that $f-g_{d}\left(L_{j, k}\right)^{d}$ is in $S$, but the degree of $f-g_{d}\left(L_{j, k}\right)^{d}$ in $y_{j}$ is less than $d$. This is a contradiction, and we get $S=\emptyset$.

Similarly to the remark before Proposition 3.8, we have $D\left(g_{d}\right)=0$. We show that every monomial appearing in $g_{d}$ does not have negative power in $x_{i}$ for each $i$. First, assume that the $i$-th component of $\varepsilon_{k, j}^{+}$is not zero. Then, it is equal to $\varepsilon_{k, j}^{i}>0$, and so $\varepsilon_{j, k}^{i}$ is negative. Hence, $\varepsilon_{l, k}^{i} \geq 0$ for any $l \neq j$ by assumption. Since $\varepsilon_{l, j}^{i}=\varepsilon_{l, k}^{i}+\varepsilon_{k, j}^{i}$, we have $0<\varepsilon_{k, j}^{i} \leq \varepsilon_{l, j}^{i}$ 
for $l \neq j$. Thus, the substitution $y_{j} \mapsto x_{i}^{-\varepsilon_{k, j}^{i}} y_{j}$ sends $f$ to $T_{j, i}(f)$. If there appeared in $g_{d}$ a monomial $\boldsymbol{x}^{a} \boldsymbol{y}^{b}$ with negative power in $x_{i}$, then $T_{j, i}(f)$ would have the monomial $\boldsymbol{x}^{a} \boldsymbol{y}^{b} y_{j}^{d}$. It also has negative power in $x_{i}$. This is a contradiction, since $T_{j, i}(f)$ is in $K[\boldsymbol{x}][\boldsymbol{y}]$ by Lemma 4.3. If the $i$-th component of $\varepsilon_{k, j}^{+}$is zero, then the expression (4.2) also implies that no monomial appearing in $g_{d}$ has negative power in $x_{i}$. Therefore, $g_{d}$ is in $K[\boldsymbol{x}][\boldsymbol{y}]$.

As a corollary to Proposition 4.4, we have the following.

COROLlaRY 4.5 (Khoury [5, Theorem 3.1]). If $m=2$, then there exist $1 \leq l \leq n$ and $1 \leq k_{j} \leq n$ with $k_{j} \neq j$ for each $j \neq l$ such that

$$
K[\boldsymbol{x}][\boldsymbol{y}]^{D}=K[\boldsymbol{x}]\left[L_{1, k_{1}}, \ldots, L_{l-1, k_{l-1}}, L_{l+1, k_{l+1}}, \ldots, L_{n, k_{n}}\right] .
$$

Proof. We prove this by induction on $n$. If $n=1$, then $K[x][y]^{D}=K[x]$ by Lemma 4.2. Hence, the assertion is true. Assume that $n>1$. Then, by change of indices if necessary, we may assume that $\delta_{1}^{1} \leq \cdots \leq \delta_{n}^{1}$. If there exist $1 \leq k<j \leq n$ such that $\delta_{k}^{2} \leq \delta_{j}^{2}$, then $\varepsilon_{j, k}^{i} \geq 0$ for $i=1,2$. Hence,

$$
K[\boldsymbol{x}][\boldsymbol{y}]^{D}=K[\boldsymbol{x}]\left[y_{1}, \ldots, y_{j-1}, y_{j+1}, \ldots, y_{n}\right]^{D}\left[L_{j, k}\right]
$$

by Proposition 4.4. Thus, the assertion follows from the induction assumption. Assume that such $k, j$ do not exist, i.e., $\delta_{n}^{2}<\cdots<\delta_{1}^{2}$. Then, $\varepsilon_{l, n-1}^{2}>0$ for any $l \neq n$. Since $\varepsilon_{n, n-1}^{1} \geq 0$, we have $K[\boldsymbol{x}][\boldsymbol{y}]^{D}=K[\boldsymbol{x}]\left[y_{1}, \ldots, y_{n-1}\right]^{D}\left[L_{n, n-1}\right]$ by Proposition 4.4. Hence, the assertion follows similarly.

Let $\phi_{1}: K[\boldsymbol{x}][\boldsymbol{y}] \rightarrow K\left[x_{2}, \ldots, x_{m}\right][\boldsymbol{y}]$ be the homomorphism which substitutes one for $x_{1}$, and $D_{1}$ the elementary $K\left[x_{2}, \ldots, x_{m}\right]$-derivation on $K\left[x_{2}, \ldots, x_{m}\right][y]$ defined by $D_{1}(f)=\phi_{1}(D(f))$ for each $f$. Then, $D_{1}$ is a monomial derivation. By definition, it follows that $\phi_{1} \circ D=D_{1} \circ \phi_{1}$ on $K[x][y]$. Recall the $\Gamma$-grading structure on $K[x][y]$ defined in Section 3. Let $\Gamma_{1}$ be the set of the images of $\left(a, l \boldsymbol{e}_{n}\right)$ in $\Gamma$ for $l \in \boldsymbol{Z}$ and $a=\left(a_{1}, \ldots, a_{m}\right) \in \boldsymbol{Z}^{m}$ with $a_{1}=0$. Then, $\Gamma_{1}$ is a subgroup of $\Gamma$, and $\bigoplus_{\gamma \in \Gamma_{1}} K[\boldsymbol{x}][\boldsymbol{y}]_{\gamma}$ is a $K\left[x_{2}, \ldots, x_{n}\right]$ subalgebra of $K[x][y]$.

Lemma 4.6. Assume that $\varepsilon_{n, j}^{1} \geq 0$ for $j=1, \ldots, n$. Then, $\phi_{1}$ induces an isomorphism

$$
\bigoplus_{\gamma \in \Gamma_{1}} K[\boldsymbol{x}][\boldsymbol{y}]_{\gamma}^{D} \rightarrow K\left[x_{2}, \ldots, x_{m}\right][\boldsymbol{y}]^{D_{1}} .
$$

PROOF. Set $R=\bigoplus_{\gamma \in \Gamma_{1}} K[\boldsymbol{x}][\boldsymbol{y}]_{\gamma}$ and $R^{\prime}=K\left[x_{2}, \ldots, x_{m}\right][\boldsymbol{y}]$. It suffices to show that $\phi_{1}$ induces an isomorphism $R \rightarrow R^{\prime}$. Indeed, it implies that $\phi_{1}\left(R^{D}\right)=\left(R^{\prime}\right)^{D_{1}}$, since $\phi_{1} \circ D=D_{1} \circ \phi_{1}$.

First, we show the injectivity. Suppose that there existed $f \in R \backslash\{0\}$ such that $\phi_{1}(f)=0$. Then, $f=\left(x_{1}-1\right) f^{\prime}$ for some $f^{\prime} \in K[\boldsymbol{x}][\boldsymbol{y}] \backslash\{0\}$. Let $p$ and $q$ be the maximal and the minimal integers $l$ with $\operatorname{deg}_{\Gamma}\left(x_{1}^{l} f^{\prime \prime}\right) \in \Gamma_{1}$ for some nonzero $\Gamma$-homogeneous component $f^{\prime \prime}$ of $f^{\prime}$, respectively. Clearly, we have $p \geq 1$ or $q \leq 0$. If $p \geq 1$, then $\operatorname{deg}_{\Gamma}\left(f^{\prime \prime}\right) \notin \Gamma_{1}$ for a $\Gamma$-homogeneous component $f^{\prime \prime}$ of $f^{\prime}$ with $\operatorname{deg}_{\Gamma}\left(x_{1}^{p} f^{\prime \prime}\right) \in \Gamma_{1}$. However, $-f^{\prime \prime}$ is a 
$\Gamma$-homogeneous component of $f$ by the maximality of $p$. Hence, $-f^{\prime \prime}$ is in $R$. This is a contradiction. Similarly, we get a contradiction if $q \leq 0$. Therefore, $\phi_{1}(f) \neq 0$ for any $f \in R \backslash\{0\}$.

For the surjectivity, it suffices to show that $\phi_{1}(R)$ contains every monomial in $R^{\prime}$. Take any monomial $\boldsymbol{x}^{a} \boldsymbol{y}^{b} \in R^{\prime}$, and put $l=\sum_{j=1}^{n} b_{j} \varepsilon_{n, j}^{1}$, where $b=\left(b_{1}, \ldots, b_{n}\right)$. Then, $l$ is nonnegative, since $\varepsilon_{n, j}^{1} \geq 0$ for all $j$ by assumption. Hence, $x_{1}^{l} \boldsymbol{x}^{a} \boldsymbol{y}^{b}$ is in $K[\boldsymbol{x}][\boldsymbol{y}]$. Note that

$$
\operatorname{deg}_{\Gamma}\left(x_{1}^{l} \boldsymbol{x}^{a} \boldsymbol{y}^{b}\right)=\operatorname{deg}_{\Gamma}\left(x_{1}^{l} \boldsymbol{x}^{a} \boldsymbol{y}^{b} \prod_{j=1}^{n}\left(\boldsymbol{x}^{\varepsilon_{j, n}} y_{j}^{-1} y_{n}\right)^{b_{j}}\right)=\operatorname{deg}_{\Gamma}\left(\boldsymbol{x}^{c} y_{n}^{\sum_{j=1}^{n} b_{j}}\right),
$$

where $c=(l, 0, \ldots, 0)+a+\sum_{j=1}^{n} b_{j} \varepsilon_{j, n}$. Since the first component of $a$ is zero, that of $c$ is equal to $l+\sum_{j=1}^{n} b_{j} \varepsilon_{j, n}^{1}=0$. Thus, $x_{1}^{l} \boldsymbol{x}^{a} \boldsymbol{y}^{b}$ is in $R$. Since $\boldsymbol{x}^{a} \boldsymbol{y}^{b}=\phi_{1}\left(x_{1}^{l} \boldsymbol{x}^{a} \boldsymbol{y}^{b}\right)$, the surjectivity is proved.

Lemma 4.7. Assume that $n=4$ and $\varepsilon_{1,3}^{1}, \varepsilon_{1,2}^{1}>0, \varepsilon_{1,4}^{1}=0$. Then, $K[\boldsymbol{x}][\boldsymbol{y}]^{D}$ is generated by $x_{1}$ and $L_{3,2}$ over $\bigoplus_{\gamma \in \Gamma_{1}} K[\boldsymbol{x}][\boldsymbol{y}]_{\gamma}^{D}$.

PROOF. Without loss of generality, we may assume that $\varepsilon_{1,3}^{1} \geq \varepsilon_{1,2}^{1}$. It suffices to show that each $\Gamma$-homogeneous element $F \in K[\boldsymbol{x}][\boldsymbol{y}]^{D}$ is written as $F=x_{1}^{p} L_{3,2}^{q} F^{\prime}$, where $p, q \in \boldsymbol{Z}_{\geq 0}$ and $F^{\prime} \in K[\boldsymbol{x}][\boldsymbol{y}]_{\gamma^{\prime}}$ for some $\gamma^{\prime} \in \Gamma_{1}$. Indeed, it also implies that $D\left(F^{\prime}\right)=0$, since $0=D(F)=x_{1}^{p} L_{3,2}^{q} D\left(F^{\prime}\right)$.

Assume that $\operatorname{deg}_{\Gamma}(F)$ is equal to the image of $\left(a, l \boldsymbol{e}_{4}\right)$, where $a=\left(a_{1}, \ldots, a_{m}\right) \in \boldsymbol{Z}^{m}$ and $l \in \boldsymbol{Z}_{\geq 0}$. We set $f=\phi(F)$. Then, $F=\tau_{\boldsymbol{x}^{a}}(f)$, as we noted before Proposition 3.4. Take any $b=\left(b_{1}, b_{2}, b_{3}, b_{4}\right) \in \operatorname{supp}(f)$. Then, by straightforward computation, we get $\tau_{\boldsymbol{x}^{a}}\left(\boldsymbol{y}^{b}\right)=\boldsymbol{x}^{c} \boldsymbol{y}^{b}$, where

$$
c=a+\left(l-b_{4}\right) \varepsilon_{4,1}+b_{2} \varepsilon_{1,2}+b_{3} \varepsilon_{1,3} .
$$

Since $\varepsilon_{4,1}^{1}=0$, the first component of $c$ is equal to $a_{1}+\tilde{l}(b)$. On the other hand, we have $\tilde{l}(b) \geq 0$, since $\varepsilon_{1,2}^{1}, \varepsilon_{1,3}^{1}>0$. Hence, $x_{1}^{-a_{1}} \boldsymbol{x}^{c} \boldsymbol{y}^{b}$ does not have negative power. Thus, $x_{1}^{-a_{1}} F$ is in $K[\boldsymbol{x}][\boldsymbol{y}]$. Clearly, $\operatorname{deg}_{\Gamma}\left(x_{1}^{-a_{1}} F\right)$ is in $\Gamma_{1}$. Therefore, if $a_{1} \geq 0$, then we are led to the desired expression $F=x_{1}^{a_{1}}\left(x_{1}^{-a_{1}} F\right)$.

Assume that $a_{1}<0$. Let $q$ be the minimal integer such that $q \varepsilon_{1,3}^{1} \geq-a_{1}$. Since the first component of (4.5) is nonnegative, we have $\tilde{l}(b) \geq-a_{1}$ for every $b \in \operatorname{supp}(f)$. Hence, $\left(y_{3}-y_{2}\right)^{q}$ divides $f$ by Lemma 3.7. It implies that $F=F^{\prime} L_{3,2}^{q}$ for some $F^{\prime} \in K[\boldsymbol{x}][\boldsymbol{y}]^{D}$. Note that $\operatorname{deg}_{\Gamma}\left(L_{3,2}^{q}\right)$ is equal to the image of $q\left(\varepsilon_{2,3}^{+}+\varepsilon_{3,4}, \boldsymbol{e}_{4}\right)$ in $\Gamma$. Hence, $\operatorname{deg}_{\Gamma}\left(F^{\prime}\right)$ is equal to that of $\left(a^{\prime},(l-q) \boldsymbol{e}_{4}\right)$, where

$$
a^{\prime}=a-q\left(\varepsilon_{2,3}^{+}+\varepsilon_{3,4}\right)=a+q \varepsilon_{1,3}-q\left(\varepsilon_{2,3}^{+}+\varepsilon_{1,4}\right) .
$$

Since the first components of $\varepsilon_{2,3}^{+}$and $\varepsilon_{1,4}$ are zero, that of $a^{\prime}$ is equal to $a_{1}+q \varepsilon_{1,3}^{1}$. By the choice of $q$, this is nonnegative. Hence, we have $F^{\prime}=x_{1}^{p} F^{\prime \prime}$ for some $p \in Z_{\geq 0}$ and $F^{\prime \prime} \in K[\boldsymbol{x}][\boldsymbol{y}]_{\gamma^{\prime}}$ with $\gamma^{\prime} \in \Gamma_{1}$, as we showed in the preceding paragraph. Therefore, we get a desired expression. 
Now, let us prove Theorem 4.1. Note that the assumption fails if and only if we can exchange the rows and columns of the matrix $\left(\delta_{i}^{j}\right)_{i, j}$ so that $\delta_{i}^{i}$ is the maximum among the components of the $i$-th column for each $i$. Under the assumption, we are reduced to one of the following two cases by such operations:

(i) $\delta_{i}^{1} \leq \delta_{1}^{1}$ and $\delta_{i}^{2} \leq \delta_{1}^{2}$ for $i=1,2,3,4$.

(ii) $\delta_{i}^{1}<\delta_{1}^{1}=\delta_{4}^{1}$ for $i=2,3$.

In fact, if we are not reduced to (ii), then there exists $1 \leq k_{j} \leq 4$ for each $j=1,2,3$ such that $\delta_{i}^{j}<\delta_{k_{j}}^{j}$ for any $i \neq k_{j}$. If further we were not reduced to (i), then $k_{j} \neq k_{l}$ for any $j \neq l$. In this case, we can exchange the rows of $\left(\delta_{i}^{j}\right)_{i, j}$ so that $k_{j}=j$ for $j=1,2,3$. This implies that $\delta_{i}^{j}<\delta_{i}^{i}$ for any $i \neq j$.

First, consider the case (i). By exchanging the row vectors $\delta_{2}, \delta_{3}$ and $\delta_{4}$ of $\left(\delta_{i}^{j}\right)_{i, j}$ if necessary, we may assume that $\delta_{4}^{3} \leq \delta_{j}^{3}$, that is, $\varepsilon_{j, 4}^{3} \geq 0$ for $j=2,3$, 4. Since $\delta_{4}^{1} \leq \delta_{1}^{1}$ and $\delta_{4}^{2} \leq \delta_{1}^{2}$ by assumption, we have $\varepsilon_{1,4}^{1}, \varepsilon_{1,4}^{2} \geq 0$. Hence, $K[\boldsymbol{x}][\boldsymbol{y}]^{D}=K[\boldsymbol{x}]\left[y_{1}, y_{2}, y_{3}\right]^{D}\left[L_{4,1}\right]$ by Proposition 4.4. Therefore, $K[\boldsymbol{x}][\boldsymbol{y}]^{D}$ is generated by $L_{2,1}, L_{3,1}, L_{3,2}$ and $L_{4,1}$ over $K[\boldsymbol{x}]$ by Corollary 3.5 .

Now, consider the case (ii). Since $\varepsilon_{2,1}^{1}, \varepsilon_{3,1}^{1}<0$ and $\varepsilon_{4,1}^{1}=0$ follow from the condition, $K[\boldsymbol{x}][\boldsymbol{y}]^{D}$ is generated by $x_{1}, L_{3,2}^{D}$ over $\bigoplus_{\gamma \in \Gamma_{1}} K[\boldsymbol{x}][\boldsymbol{y}]_{\gamma}^{D}$ by Lemma 4.7. By Lemma 4.6, $\bigoplus_{\gamma \in \Gamma_{1}} K[\boldsymbol{x}][\boldsymbol{y}]_{\gamma}^{D}$ is isomorphic to $K\left[x_{2}, x_{3}\right][\boldsymbol{y}]^{D^{\prime}}$ via $\phi_{1}$, since $\varepsilon_{4, j}^{1} \geq 0$ for any $j$. Then, by Corollary 4.5, there exist $1 \leq l \leq 4$, and $1 \leq k_{i} \leq 4$ with $k_{i} \neq i$ for $i \in\{1,2,3,4\} \backslash\{l\}$ such that $K\left[x_{2}, x_{3}\right][y]^{D^{\prime}}$ is generated by $L_{k_{i}, i}^{D^{\prime}}$ for $i \in\{1,2,3,4\} \backslash\{l\}$ over $K\left[x_{2}, x_{3}\right]$. Since $\phi_{1}\left(L_{i, j}^{D}\right)=L_{i, j}^{D^{\prime}}$ for $i, j$, the $K\left[x_{2}, x_{3}\right]$-algebra $\bigoplus_{\gamma \in \Gamma_{1}} K[\boldsymbol{x}][\boldsymbol{y}]_{\gamma}^{D}$ is generated by $L_{k_{i}, i}^{D}$ for $i \in\{1,2,3,4\} \backslash\{l\}$. Therefore, $K[\boldsymbol{x}][\boldsymbol{y}]^{D}$ is generated by $L_{3,2}^{D}$ and $L_{k_{i}, i}^{D}$ for $i \in\{1,2,3,4\} \backslash\{l\}$ over $K[x]$. This completes the proof of Theorem 4.1 .

Let $D$ be any elementary monomial $K[\boldsymbol{x}]$-derivation on $K[\boldsymbol{x}][\boldsymbol{y}]$ for $(m, n)=(3,4)$. By Theorems 1.4 and 4.1, we settled the problem of finite generation of $K[\boldsymbol{x}][\boldsymbol{y}]^{D}$ except in the case $\varepsilon_{i, j}^{i}>0$ for any $i \neq j$ and $\xi(D)>1$.

Conjecture 4.8. Assume that $(m, n)=(3,4)$, and $\varepsilon_{i, j}^{i}>0$ for any $i \neq j$. If $\xi(D)>1$, then $K[\boldsymbol{x}][\boldsymbol{y}]^{D}$ is finitely generated.

Note that the conjecture is true if there exist distinct $r, s \in\{1,2,3\}$ such that $\xi_{r}(D) \geq 1$ and $\xi_{s}(D) \geq 1$. We show this for $(r, s)=(2,3)$. The conditions $\xi_{2}(D) \geq 1$ and $\xi_{3}(D) \geq 1$ imply, respectively, that $\varepsilon_{3,4}^{2} \geq 0$ or $\varepsilon_{1,4}^{2} \geq 0$, and $\varepsilon_{1,4}^{3} \geq 0$ or $\varepsilon_{2,4}^{3} \geq 0$. Furthermore, we have $\varepsilon_{1,4}^{1}>0, \varepsilon_{2,4}^{2}>0$ and $\varepsilon_{3,4}^{3}>0$ by assumption. Hence, for each $i=1,2,3$, we have $\varepsilon_{1,4}^{i} \geq 0$ or $\varepsilon_{l, 4}^{i} \geq 0$ for $l=2,3,4$. Thus, $K[\boldsymbol{x}][\boldsymbol{y}]^{D}=K[\boldsymbol{x}]\left[y_{2}, y_{3}, y_{4}\right]^{D}\left[L_{4,1}\right]$ by Proposition 4.4. Therefore, $K[\boldsymbol{x}][\boldsymbol{y}]^{D}$ is generated by $L_{3,2}, L_{4,1}, L_{4,2}$ and $L_{4,3}$ over $K[\boldsymbol{x}]$ by Corollary 3.5.

There exists an example of an elementary monomial $K[x]$-derivation on $K[x][y]$ for $(m, n)=(3,4)$ whose kernel is finitely generated, and $\xi_{i}(D)<1$ for $i=1,2,3$. Kurano [7] showed that the kernel of $D_{1,3}$ is finitely generated. In fact, he showed that it is generated by 
$x_{1}, x_{2}, x_{3}, L_{i, j}$ for $(i, j) \in \boldsymbol{Z} \times \boldsymbol{Z}$ with $1 \leq j<i \leq 4$ and

$$
x_{i} y_{4}^{2}-2 x_{j} x_{k} y_{i} y_{4}+x_{i} x_{k}^{2} y_{i} y_{j}+x_{i} x_{j}^{2} y_{i} y_{k}-x_{i}^{3} y_{j} y_{k}
$$

for $(i, j, k)=(1,2,3),(2,3,1),(3,1,2)$ over $K$. Moreover, [7, Lemma 3.2] implies that the set of these polynomials is a SAGBI basis for the lexicographic order $\preceq_{\text {lex }}$ with (3.3). For this derivation, we have $\xi_{i}\left(D_{1,3}\right)=1 / 2$ for $i=1,2,3$.

\section{REFERENCES}

[ 1 ] D. Daigle and G. Freudenburg, A counterexample to Hilbert's fourteenth problem in dimension 5, J. Algebra 221 (1999), 528-535.

[2] J. Deveney And D. Finston, $G_{a}$-actions on $\boldsymbol{C}^{3}$ and $\boldsymbol{C}^{7}$, Comm. Algebra 22 (1994), 6295-6302.

[3] A. VAn DEN Essen, Polynomial automorphisms and the Jacobian conjecture, Progress in Mathematics, Vol. 190, Birkhäuser, Basel, Boston, Berlin, 2000.

[ 4 ] G. Freudenburg, A counterexample to Hilbert's fourteenth problem in dimension six, Transform. Groups $5(2000), 61-71$

[ 5 ] J. KhouRY, On some properties of elementary monomial derivations in dimension six, J. Pure Appl. Algebra 156 (2001), 69-79

[ 6] H. Kojima And M. MiYanishi, On Roberts' counterexample to the fourteenth problem of Hilbert, J. Pure Appl. Algebra 122 (1997), 277-292.

[ 7 ] K. KURAnO, Positive characteristic finite generation of symbolic Rees algebra and Roberts' counterexamples to the fourteenth problem of Hilbert, Tokyo J. Math. 16 (1993), 473-496.

[8] S. KURODA, The infiniteness of the SAGBI bases for certain invariant rings, Osaka J. Math. 39 (2002), 665680

[ 9] S. KurodA, A condition for finite generation of the kernel of a derivation, J. Algebra 262 (2003), 391-400.

[10] M. MiYanishi, Lectures on Curves on Rational and Unirational Surfaces, Tata Institute of Fundamental Research, Springer, Berlin, 1978

[11] S. Mukai, Counterexample to Hilbert's fourteenth problem for the 3-dimensional additive group, Preprint 1343, Research Institute for Mathematical Sciences, Kyoto University, 2001.

[12] M. NAGATA, Lectures on the fourteenth problem of Hilbert, Tata Institute of Fundamental Research, Bombay, 1965.

[13] L. Robbiano And M. Sweedler, Subalgebra bases, in Commutative Algebra (W. Bruns and A. Simis, eds.) 61-87, Lecture Notes in Math. 1430, Springer, Berlin, Heidelberg, New York, Tokyo, 1988

[14] P. RoberTs, An infinitely generated symbolic blow-up in a power series ring and a new counterexample to Hilbert's fourteenth problem, J. Algebra 132 (1990), 461-473.

RESEARCH InSTITUTE FOR MATHEMATICAL SCIENCES

KYOTO UNIVERSITY

KYOTOI 606-8502

JAPAN

E-mail address: kuroda@kurims.kyoto-u.ac.jp 